\title{
MODERATE DEVIATIONS FOR THE SPECTRAL MEASURE OF CERTAIN RANDOM MATRICES
}

\author{
A. DEMBO ${ }^{\mathrm{a}, 1}$, A. GUIONNET ${ }^{\mathrm{b}, *, 2}$, O. ZEITOUNI ${ }^{\mathrm{c}, 3}$ \\ ${ }^{a}$ Departments of Mathematics and of Statistics, Stanford University, Stanford, CA 94305, USA \\ ${ }^{\mathrm{b}}$ UMPA, École normale superieure de Lyon, 46, allée d'Italie, 69364 Lyon cedex 07, France \\ ${ }^{\mathrm{c}}$ Departments of Electrical Engineering and of Mathematics, Technion, Haifa 32000, Israel
}

Received 14 February 2002, accepted 25 February 2003

ABSTRACT. - We derive a moderate deviations principle for matrices of the form $X_{N}=$ $D_{N}+W_{N}$ where $W_{N}$ are Wigner matrices and $D_{N}$ is a sequence of deterministic matrices whose spectral measures converge in a strong sense to a limit $\mu_{D}$. Our techniques are based on a dynamical approach introduced by Cabanal-Duvillard and Guionnet.

(c) 2003 Éditions scientifiques et médicales Elsevier SAS

MSC: 60F99; 15A52

Keywords: Limit theorems; Random matrices

RÉSUMÉ. - Nous démontrons un principe de déviations modérées pour la mesure spectrale de matrices de la forme $X_{N}=D_{N}+W_{N}$ où $W_{N}$ sont des matrices de Wigner et $D_{N}$ une suite de matrices déterministes dont la mesure spectrale converge fortement vers une loi limite $\mu_{D}$. Nous utilisons pour cela des techniques basées sur l'approche dynamique introduite par CabanalDuvillard et Guionnet.

๑) 2003 Éditions scientifiques et médicales Elsevier SAS

\section{Introduction}

Let $\mathcal{M}_{N}$ denote the set of $N \times N$ Hermitian matrices, and let $W_{N} \in \mathcal{M}_{N}$ be a Gaussian Wigner matrix, that is, a symmetric or Hermitian matrix with real (respectively, complex) i.i.d. Gaussian entries of covariance $N^{-1}$ above the diagonal. We consider

$$
X_{N}=D_{N}+W_{N}
$$

\footnotetext{
* Corresponding author.

E-mail address: aguionne@umpa.ens-lyon.fr (A. Guionnet).

${ }^{1}$ Research partially supported by NSF grant \#DMS-0072331.

2 Research partially supported by NSF grant \#DMS-9631278.

${ }^{3}$ Research partially supported by a grant from the Israel Science Foundation, administered by the Israel Academy of Sciences.
} 
for a diagonal matrix $D_{N}$ with diagonal elements $d_{i}^{N}$ and spectral measure $\hat{\mu}_{D_{N}}^{N}:=$ $N^{-1} \sum_{i=1}^{N} \delta_{d^{N}}$ converging towards a compactly supported probability measure $\mu_{D}$, in such a way that

$$
\tilde{c}(\varepsilon):=\max _{i=1,2} \max _{N} \sup _{z \in \mathbb{C} \backslash \mathbb{R},|\Im(z)| \geqslant \varepsilon} N\left|\operatorname{tr}_{N}\left(z-D_{N}\right)^{-i}-\int(z-x)^{-i} d \mu_{D}(x)\right|<\infty \quad \forall \varepsilon>0
$$

(where $\operatorname{tr}_{N}$ denotes the trace of the matrix, normalized by its size $N$ ).

Denote by $\hat{\mu}_{X_{N}}^{N}$ the spectral measure of $X_{N}$. Recall (see, e.g., [19]) that $\hat{\mu}_{X_{N}}^{N}$ converges weakly to the compactly supported probability measure $\mu_{1}^{*}$ where $\mu_{t}^{*}=\mu_{D} \boxplus \sigma_{t}, \sigma_{t}$ denoting the Wigner semi-circular distribution $\sigma_{t}(d x)=(2 \pi t)^{-1} \sqrt{4 t-x^{2}} d x$ and $\boxplus$ denoting free convolution of measures.

Large deviations (in the scale $N^{2}$ ) and CLT's for $\hat{\mu}_{X_{N}}^{N}$ are obtained in $[5,6,9,12]$, by a dynamical approach based on the observation that $W_{N}$ can be constructed as a Hermitian or symmetric Brownian motion at time one. These large deviations are essential tools in the study of so-called "matrix models" in physics, see [10]. It is our goal in this work to extend this analysis to study moderate deviations of $\hat{\mu}_{X_{N}}^{N}$. Note that since exponentially good approximations at the scale $N^{2}$ are no longer such for the moderate deviation scales considered here, this study is far from being a straight forward extension of the previous analysis mentioned above. Our work can be considered as a non-commutative partial analogue of the moderate deviations principle for the empirical measure of i.i.d. random variables, see [21].

In order to state our results, we first introduce some notations. Let $\mathcal{S}$ tieljes $(\mathbb{C})$ be the complex vector space generated by the Stieljes functions $\left\{f(x)=(z-x)^{-1}, z \in \mathbb{C} \backslash \mathbb{R}\right\}$ (with $x \in \mathbb{R}$ ), and denote by $\mathcal{S}$ tieljes $(\mathbb{R}) \subset \mathcal{C}_{b}^{\infty}(\mathbb{R})$ the subset of real valued functions in $\mathcal{S}$ tieljes $(\mathbb{C})$. Note that $\Re(z-x)^{-1} \in \mathcal{S}$ tieljes $(\mathbb{R})$ for $z \in \mathbb{C} \backslash \mathbb{R}$.

To any $f=f_{1} \in \mathcal{S}$ tieljes $(\mathbb{C})$, we associate the function $s \mapsto f_{s}$ that solves the differential equation

$$
\partial_{s} f_{s}(x)=-\int \frac{\partial_{x} f_{s}(x)-\partial_{x} f_{s}(y)}{x-y} d \mu_{s}^{*}(y), \quad f_{1}(x)=f(x) .
$$

In Section 4 below we show that (1.1) has a unique solution whenever $f(x)=c(z-x)^{-1}$, $z \in \mathbb{C} \backslash \mathbb{R}$ (given by (4.1) and (4.2)). By the linearity of (1.1), the same applies for any $f \in \mathcal{S}$ tieljes $(\mathbb{C})$.

For any $f, g \in \mathcal{S}$ tieljes $(\mathbb{R})$ and the corresponding solutions $f_{s}, g_{s}$ of (1.1), define

$$
\mathcal{V}_{t}(f, g)=\int_{0}^{t} \int_{0}\left(\partial_{x} f_{s}\right)(x)\left(\partial_{x} g_{s}\right)(x) d \mu_{s}^{*}(x) d s .
$$

In what follows, we let $\mathcal{S}$ tieljes' $(\mathbb{R}):=\left\{f^{\prime}: f \in \mathcal{S}\right.$ tieljes $\left.(\mathbb{R})\right\}$ and $L_{c}^{p}(\mathbb{R})$ denote the subset of $L^{p}(\mathbb{R})$ consisting of functions of compact support. Let

$$
F_{N}(x):=\mu_{1}^{*}((-\infty, x])-\hat{\mu}_{X_{N}}^{N}((-\infty, x]),
$$


which is in $L_{c}^{p}\left(\mathbb{R}\right.$ ) for any $p \geqslant 1$ whenever $\mu_{D}$ (and hence $\mu_{1}^{*}$ ) is of compact support. Integration by parts shows that for all $f \in \mathcal{C}_{b}^{1}(\mathbb{R})$,

$$
X_{N}(f):=\int f(x) d \hat{\mu}_{X_{N}}^{N}(x)-\int f(x) d \mu_{1}^{*}(x)=\int f^{\prime}(x) F_{N}(x) d x .
$$

With some abuse of notation we let $\langle g, G\rangle$ denote the value of a linear functional $G$ at $g$ which is in a vector subspace of $\mathcal{C}_{b}(\mathbb{R})$ (to be understood from the context of the statement), using also $\langle g, G\rangle=\int g(x) G(x) d x$ in the case $G \in L^{1}(\mathbb{R})$.

Our main result now reads

THEOREM 1.1. - For any $a_{N} \rightarrow 0$ such that $N a_{N} \rightarrow \infty$, the sequence of random variables $\left\{a_{N}^{-1} F_{N}\right\}_{N}$ in $L_{c}^{1}(\mathbb{R})$ equipped with the Stieljes' $(\mathbb{R})$-topology and the corresponding cylinder $\sigma$-field, satisfies the Large Deviation Principle (LDP) with speed $\left(N a_{N}\right)^{-2}$ and good rate function $I(\cdot)$ defined by

$$
I(F):=\frac{\beta}{2} \sup _{h \in \mathcal{S} \text { tieljes }(\mathbb{R})}\left\{\left\langle h^{\prime}, F\right\rangle-\frac{1}{2} \mathcal{V}_{1}(h, h)\right\}
$$

with $\beta=1$ (resp. $\beta=2$ ) in the symmetric (resp. Hermitian) case.

(We refer to [8] for standard terminology concerning the LDP. Because the rate function is the same for a range of speeds, we refer to the LDP in Theorem 1.1 as a moderate deviation principle (MDP).)

Note that the topology for the MDP in Theorem 1.1 is weaker than the $\mathcal{C}_{b}(\mathbb{R})$-topology of convergence in law. Some strengthening of the former topology can be achieved by considering the $N$-dependent centering

$$
\bar{F}_{N}(x):=\mathbb{E} \hat{\mu}_{X_{N}}^{N}((-\infty, x])-\hat{\mu}_{X_{N}}^{N}((-\infty, x]) .
$$

Specifically, with $\mathcal{C}_{b}^{\prime}(\mathbb{R}):=\left\{f^{\prime}: f \in \mathcal{C}_{b}^{1}(\mathbb{R})\right\}$ denoting the space of bounded continuous functions which possess a bounded primitive, we have:

THEOREM 1.2. - For any $a_{N} \rightarrow 0$ such that $N a_{N} \rightarrow \infty$, the sequence of random variables $\left\{a_{N}^{-1} \bar{F}_{N}\right\}_{N}$ in $L_{c}^{1}(\mathbb{R})$ equipped with the $\mathcal{C}_{b}^{\prime}(\mathbb{R})$-topology and the corresponding cylinder $\sigma$-field, satisfies the LDP with speed $\left(N a_{N}\right)^{-2}$ and good rate function I $(\cdot)$ of (1.3).

As the rate function $I(\cdot)$ is not particularly transparent to work with, we provide next some useful information about it. First, it follows from the CLT of [9, Section 6] that for any $h \in \mathcal{S}$ tieljes $(\mathbb{R})$,

$$
\mathcal{V}_{1}(h, h)=\lim _{N \rightarrow \infty} N^{2} \mathbb{E}\left(\bar{X}_{N}(h)^{2}\right)=\lim _{N \rightarrow \infty} \operatorname{Var}\left(N X_{N}(h)\right) .
$$

Our proof of Theorems 1.1 and 1.2 provides also that for any $a_{N} \rightarrow 0$ such that $N a_{N} \rightarrow \infty$,

$$
\begin{aligned}
\mathcal{V}_{1}(h, h) & =2 \lim _{N \rightarrow \infty}\left(N a_{N}\right)^{-2} \log \mathbb{E}\left(\mathrm{e}^{N^{2} a_{N} \bar{X}_{N}(h)}\right) \\
& =2 \lim _{N \rightarrow \infty}\left(N a_{N}\right)^{-2} \log \mathbb{E}\left(\mathrm{e}^{N^{2} a_{N} X_{N}(h)}\right) .
\end{aligned}
$$


Recall that $\mu_{1}^{*}$ has a density $p_{1}$ (see [2, Corollary 2]), and for $F=f p_{1}$ with $f \in L^{2}\left(\mu_{1}^{*}\right)$ set

$$
J(F):=\frac{\beta}{4} \int f^{2}(x) d \mu_{1}^{*}(x)+\frac{\beta}{4} \iint\left(\frac{f(x)-f(y)}{x-y}\right)^{2} d \mu_{1}^{*}(x) d \mu_{1}^{*}(y)
$$

(which is well defined, though possibly infinite), setting $J(F)=\infty$ for all $F \in L_{c}^{1}(\mathbb{R})$ not of the above form. Let $L_{c}^{1, *}(\mathbb{R})$ denote the subset of $L_{c}^{1}(\mathbb{R})$ consisting of functions whose support is contained in the support of $\mu_{1}^{*}$. In Section 6 we prove that

LEMMA 1.3. - The function $I(\cdot)$ is finite only for linear functionals on $\mathcal{S}$ tieljes' $(\mathbb{R})$ that are of the form $\left\langle h^{\prime}, F\right\rangle=\int F(x) h^{\prime}(x) d x$ for some $F \in L_{c}^{1, *}(\mathbb{R})$, in which case

$$
\frac{\beta}{4}\left(\int|F(x)| d x\right)^{2} \leqslant \frac{\beta}{4} \int \frac{F(x)^{2}}{p_{1}(x)} d x \leqslant I(F) .
$$

Further, $I(F) \leqslant J(F)$ for $F=f p_{1}$ with $f \in \mathcal{C}_{b}^{1}(\mathbb{R})$, and more generally, for all

$$
\begin{gathered}
F \in \mathcal{P}:=\left\{f p_{1}: f \in L^{2}\left(\mu_{1}^{*}\right), \exists Q^{\delta} \text { polynomials such that } Q^{\delta} \underset{\delta \rightarrow 0}{\mathcal{L}} f,\right. \\
\left.\liminf _{\delta \rightarrow 0} J\left(Q^{\delta} p_{1}\right) \leqslant J\left(f p_{1}\right)\right\} .
\end{gathered}
$$

Here, $Q^{\delta} \underset{\delta \rightarrow 0}{\stackrel{\mathcal{L}}{\longrightarrow}} f$ iff $\int g(x) Q^{\delta}(x) p_{1}(x) d x$ converges towards $\int g(x) f(x) p_{1}(x) d x$ for any bounded continuous function $g$ on $\mathbb{R}$.

In the special case $\mu_{D}=0$, one can make the rate function more explicit. Indeed, in this case $\mu_{t}^{*}=\sigma_{t}$ and $p_{1}(y)=(2 \pi)^{-1} \sqrt{4-y^{2}}$, and one obtains

Lemma 1.4. - Suppose $\mu_{D}=0$. Then, for every $h \in \mathcal{S}$ tieljes $(\mathbb{R})$,

$$
\mathcal{V}_{1}(h, h)=-\frac{1}{2 \pi^{2}} \int_{-2}^{2} \int_{-2}^{2} \frac{h(x) h^{\prime}(y)}{y-x} \frac{\sqrt{4-y^{2}}}{\sqrt{4-x^{2}}} d y d x .
$$

Moreover, assume $F=f p_{1} \in L_{c}^{1}(\mathbb{R})$ for some $f \in \mathcal{C}_{b}^{3}(\mathbb{R})$. Then

$$
I(F)=J(F)=-\frac{\beta}{2} \int_{-2}^{2} \int_{-2}^{2} F^{\prime}(x) F^{\prime}(y) \log |x-y| d x d y .
$$

The expression in the right-hand side of (1.9) resembles Voiculescu's non-commutative entropy of $D_{N}+W_{N}$ taken at the measure $F^{\prime}(x) d x$.

The structure of the article is as follows. In Section 2, we introduce the (matrix valued) Brownian motion $W_{N}(t)$ and recall the elements of stochastic calculus we need. Section 3 is devoted to the proof of a CLT type approximation for the empirical Stieljes transform $M_{t}^{N}(z)$ of $X_{N}(t)=D_{N}+W_{N}(t)$. Section 4 controls the influence of other centerings on the convergence properties of $M_{t}^{N}(z)$. Our moderate deviations results are proved in Section 5. We present first in Theorem 5.1 a finite-dimensional moderate de- 
viations principle (for $X_{N}\left(f^{(i)}\right), i=1, \ldots, d$ ) which among other things implies (1.5), and then the projective limits argument leading to Theorems 1.1 and 1.2, deferring the proofs of Lemma 1.3 (via free probability theory) and Lemma 1.4 to Section 6.

\section{Itô's calculus}

Let $H_{N}(\cdot)$ (respectively, $S_{N}(\cdot)$ ) be a $N \times N$ Hermitian (respectively, symmetric) Brownian motion constructed via independent real valued Brownian motions $\left(\beta_{i, j}, \tilde{\beta}_{k, l}\right)_{1 \leqslant i \leqslant j \leqslant N}^{1 \leqslant k<1 \leqslant N}$ by

$$
H_{N}^{k, l}= \begin{cases}\frac{1}{\sqrt{2 N}}\left(\beta_{k, l}+i \tilde{\beta}_{k, l}\right) & \text { if } k<l, \\ \frac{1}{\sqrt{2 N}}\left(\beta_{l, k}-i \tilde{\beta}_{l, k}\right) & \text { if } k>l \\ \frac{1}{\sqrt{N}} \beta_{l, l} & \text { if } k=l\end{cases}
$$

and

$$
S_{N}^{k, l}=\frac{\sqrt{1+\delta_{k=l}}}{\sqrt{N}} \beta_{k \wedge l, k \vee l},
$$

respectively. Take $W_{N}(t)=H_{N}(t)$ in the Hermitian case and $W_{N}(t)=S_{N}(t)$ in the symmetric case. Then, $W_{N}(1)$ is a complex (respectively, real), Wigner matrix. Let $\hat{\mu}_{t}^{N}$ denote the spectral measure of $X_{N}(t)=D_{N}+W_{N}(t)$ (note that $\hat{\mu}_{1}^{N}=\hat{\mu}_{X_{N}}^{N}$ ), then $\hat{\mu}_{t}^{N}$ can be studied by use of Itô's calculus as we now explain.

It was proved in $[3,6]$ that $\hat{\mu}^{N}$ satisfies an Itô's formula (in the special case where $\hat{\mu}_{D}^{N}=\delta_{0}$, assumption which is in fact clearly irrelevant). Then, if we denote, for any $f, g \in \mathcal{C}_{b}^{2,1}(\mathbb{R} \times[0,1])$, any $s \leqslant t \in[0,1]$, and any $\nu, \in \mathcal{C}([0,1], \mathcal{P}(\mathbb{R}))$,

$$
\begin{aligned}
S^{s, t}(v, f)= & \int f(x, t) d v_{t}(x)-\int f(x, s) d v_{s}(x)-\int_{s}^{t} \int_{s} \partial_{u} f(x, u) d v_{u}(x) d u \\
& -\frac{1}{2} \iiint_{s}^{t} \frac{\partial_{x} f(x, u)-\partial_{x} f(y, u)}{x-y} d v_{u}(x) d v_{u}(y) d u
\end{aligned}
$$

and

$$
\langle f, g\rangle_{s, t}^{\nu}=\int_{s}^{t} \int_{x} \partial_{x} f(x, u) \partial_{x} g(x, u) d v_{u}(x) d u,
$$

we have

THEOREM 2.1 [6]. - In the Hermitian case, for any $N \in \mathbb{N}$, any $f \in \mathcal{C}_{b}^{2,1}(\mathbb{R} \times[0,1])$ and any $s \in[0,1),\left(S^{s, t}\left(\hat{\mu}^{N}, f\right), s \leqslant t \leqslant 1\right)$ is a bounded continuous martingale with quadratic variation

$$
\left\langle S^{s, \cdot}\left(\hat{\mu}^{N}, f\right)\right\rangle_{t}=\frac{1}{N^{2}}\langle f, f\rangle_{s, t}^{\hat{\mu}^{N}}
$$


In the symmetric case, for any $N \in \mathbb{N}$, any $f \in \mathcal{C}_{b}^{2,1}(\mathbb{R} \times[0,1])$ and any $s \in[0,1)$, $\left(\widetilde{S}^{s, t}\left(\hat{\mu}^{N}, f\right):=S^{s, t}\left(\hat{\mu}^{N}, f\right)-(2 N)^{-1} \int_{s}^{t} \int f^{\prime \prime}(x) d \hat{\mu}_{u}^{N}(x) d u, s \leqslant t \leqslant 1\right)$ is a bounded continuous martingale with quadratic variation

$$
\left\langle\widetilde{S}^{s, \cdot}\left(\hat{\mu}^{N}, f\right)\right\rangle_{t}=\frac{2}{N^{2}}\langle f, f\rangle_{s, t}^{\hat{\mu}^{N}} .
$$

Note that it is not hard to see (see [6] or [11]) that the law of $\hat{\mu}^{N}$ is tight in both Hermitian and symmetric settings. The limit points are characterized by

$$
S^{s, t}\left(\mu^{*}, f\right)=0
$$

for all functions $f \in \mathcal{C}_{b}^{2,1}(\mathbb{R} \times[0,1])$. It can be shown (see [6, Corollary 1.4] or [12]) that such an equation has a unique solution $\mu_{\text {. }}^{*}$, given by the free convolution $\mu_{t}^{*}=\mu_{D} \boxplus \sigma_{t}$.

In the sequel, we shall be interested in specific test functions of the Stieljes type:

$$
f(x, t)=\frac{c_{t}}{z_{t}-x}
$$

with a complex-valued differentiable function $z:[0,1] \rightarrow \mathbb{C} \backslash \mathbb{R}$ with non-vanishing imaginary part and a complex-valued differentiable function $c_{t}:[0,1] \rightarrow \mathbb{C}$. Observe that in this case, for any $v \in \mathcal{C}([0,1], \mathcal{P}(\mathbb{R}))$,

$$
\begin{aligned}
& \frac{1}{2} \iint \frac{\partial_{x} f(x, u)-\partial_{x} f(y, u)}{x-y} d v_{u}(x) d v_{u}(y) \\
& \quad=c_{u} \int \frac{1}{z_{u}-x} d v_{u}(x) \int \frac{1}{\left(z_{u}-x\right)^{2}} d v_{u}(x) .
\end{aligned}
$$

\section{Central limit approximation}

Following Israelsson (see [13, Proposition 1]), we prove the following central limit type approximation. Throughout, we set $\beta=1$ in the symmetric case and $\beta=2$ in the Hermitian case.

LEMmA 3.1. - Consider Hermitian or symmetric matrices such that (A) holds. Then, for any $\eta>0$, there exists a finite constant $C(\eta)$ such that for all $N$ and $z \in \mathbb{C} \backslash \mathbb{R}, z=$ $a+\mathrm{i} b,|b| \geqslant \eta$,

$$
\max _{j=1,2} \sup _{\tau \in[0,1]} \mathbb{E}\left[\left|\operatorname{tr}_{N}\left(z-X_{N}(\tau)\right)^{-j}-\int(z-x)^{-j} d \mu_{\tau}^{*}(x)\right|^{2}\right]^{1 / 2} \leqslant \frac{C(\eta)}{N} .
$$

Proof. - Israelsson [13] considers only the symmetric case with Ornstein-Uhlenbeck entries. Hence, for completeness, we next adapt his approach to the context of the lemma. The main idea, used also by [5] and [9] is to choose $(c ., z$.$) in such a way that the$ finite variation term in Theorem 2.1 is negligible. Whereas Cabanal-Duvillard [5] and Guionnet [9] choose a non-random $(c, z$.) that is independent of $N$ and then control 
the remainder term of finite variation, we follow Israelsson [13] in choosing $(c ., z$.) randomly and depending on $N$ in such a way that this term completely vanishes.

We first consider the Hermitian case and $j=1$ in (3.1). For $z \in \mathbb{C} \backslash \mathbb{R}$ denote

$$
M_{t}^{N}(z)=\operatorname{tr}_{N}\left(z \mathbf{I}-X_{N}(t)\right)^{-1}, \quad M_{t}(z)=\int(z-x)^{-1} d \mu_{t}^{*}(x) .
$$

Applying Theorem 2.1 to $f(x, t)$ of (2.5), and using (2.6) it is easy to check that for any continuously differentiable functions $\left(c, z\right.$.) such that $z_{t}$ stays uniformly away from the real axis,

$$
\begin{aligned}
c_{t} M_{t}^{N}\left(z_{t}\right)= & c_{0} M_{0}^{N}\left(z_{0}\right)+\int_{0}^{t}\left[\left(\partial_{s} c_{s}\right) M_{s}^{N}\left(z_{s}\right)+c_{s}\left(\partial_{s} z_{s}\right) \partial_{z} M_{s}^{N}\left(z_{s}\right)\right. \\
& \left.-c_{s} M_{s}^{N}\left(z_{s}\right) \partial_{z} M_{s}^{N}\left(z_{s}\right)\right] d s+m_{t}^{N}(z, c .)
\end{aligned}
$$

with the bounded, complex valued, martingale $m^{N}(z, c$.) having the quadratic variations

$$
\begin{aligned}
& \left\langle\Re\left(m^{N}(z, c .)\right)\right\rangle_{t}=\frac{1}{N^{2}} \int_{0}^{t} \int_{0} \Re\left(\frac{c_{u}}{\left(z_{u}-x\right)^{2}}\right)^{2} d \hat{\mu}_{u}^{N}(x) d u, \\
& \left\langle\Im\left(m^{N}(z ., c .)\right)\right\rangle_{t}=\frac{1}{N^{2}} \int_{0}^{t} \int\left(\frac{c_{u}}{\left(z_{u}-x\right)^{2}}\right)^{2} d \hat{\mu}_{u}^{N}(x) d u .
\end{aligned}
$$

Since $|z-x| \geqslant|\Im(z)|$ for $x \in \mathbb{R}$, it follows that $M_{t}^{N}(\cdot)$ and $M_{t}(\cdot)$ are uniformly Lipschitz continuous on $\mathbb{C} \backslash \mathbb{R} \times[-|b|,|b|]$. Specifically, there

$$
\left|M_{t}^{N}(z)-M_{t}^{N}(\tilde{z})\right| \vee\left|M_{t}(z)-M_{t}(\tilde{z})\right| \leqslant|b|^{-2}|z-\tilde{z}| .
$$

Fixing $\tau \in[0,1]$, following Israelsson [13], we choose $(c ., z)=.\left(c_{.}^{N}, z^{N}\right)$ to be the solution of

$$
\begin{aligned}
& \partial_{t} z_{t}^{N}=\frac{1}{2}\left(M_{t}^{N}\left(z_{t}^{N}\right)+M_{t}\left(z_{t}^{N}\right)\right), \quad z_{\tau}^{N}=z, \\
& \partial_{t} c_{t}^{N}=\frac{1}{2}\left(\partial_{z} M_{t}^{N}\left(z_{t}^{N}\right)+\partial_{z} M_{t}\left(z_{t}^{N}\right)\right) c_{t}^{N}, \quad c_{\tau}^{N}=1,
\end{aligned}
$$

whose existence and uniqueness we next prove. Indeed, the sign of $\Im\left(M_{t}^{N}(\xi)+M_{t}(\xi)\right)$ is opposite to that of $\Im(\xi)$. Thus, taking $u_{t}^{(l)}, l=0,1, \ldots$, such that $u_{\tau}^{(l)}=z$ for all $l, u_{t}^{(0)}=z$ for $t \in[0, \tau]$ and

$$
\partial_{t} u_{t}^{(l+1)}=\frac{1}{2}\left(M_{t}^{N}\left(u_{t}^{(l)}\right)+M_{t}\left(u_{t}^{(l)}\right)\right),
$$

it is easy to see by induction that $\left|\Im\left(u_{t}^{(l)}\right)\right| \geqslant|b|$ for all $t \in[0, \tau]$ and $l \geqslant 0$. The uniform Lipschitz property of $M_{t}^{N}(\cdot)+M_{t}(\cdot)$ implies by Gronwall's lemma that the sequence $u^{(l)}$ converges uniformly on $[0, \tau]$ to the unique solution of $(3.4)$. The existence of a 
unique solution of (3.5) is then clear. Note that $t \mapsto\left|\Im\left(z_{t}^{N}\right)\right|$ is monotone non-increasing on $[0, \tau]$. Moreover, by (2.4), it is easy to check that for $t \in[0, \tau]$,

$$
\begin{aligned}
c_{t}^{N} M_{t}\left(z_{t}^{N}\right)= & c_{0}^{N} M_{0}\left(z_{0}^{N}\right)+\int_{0}^{t}\left[-c_{s}^{N} M_{s}\left(z_{s}^{N}\right) \partial_{z} M_{s}\left(z_{s}^{N}\right)+\left(\partial_{s} c_{s}^{N}\right) M_{s}\left(z_{s}^{N}\right)\right. \\
& \left.+c_{s}^{N}\left(\partial_{s} z_{s}^{N}\right) \partial_{z} M_{s}\left(z_{s}^{N}\right)\right] d s \\
= & c_{0}^{N} M_{0}\left(z_{0}^{N}\right)+\int_{0}^{t} \frac{c_{s}^{N}}{2}\left(M_{s}\left(z_{s}^{N}\right) \partial_{z} M^{N}\left(z_{s}^{N}\right)+M_{s}^{N}\left(z_{s}^{N}\right) \partial_{z} M\left(z_{s}^{N}\right)\right) d s,
\end{aligned}
$$

where (3.6) is a consequence of (3.4) and (3.5). Similarly, we find that

$$
\begin{aligned}
c_{t}^{N} M_{t}^{N}\left(z_{t}^{N}\right)= & c_{0}^{N} M_{0}^{N}\left(z_{0}^{N}\right)+\int_{0}^{t} \frac{c_{s}^{N}}{2}\left(M_{s}^{N}\left(z_{s}^{N}\right) \partial_{z} M\left(z_{s}^{N}\right)+M_{s}\left(z_{s}^{N}\right) \partial_{z} M^{N}\left(z_{s}^{N}\right)\right) d s \\
& +m_{t}^{N}\left(z_{.}^{N}, c^{N}\right) .
\end{aligned}
$$

Subtracting (3.6) from (3.7) we find that

$$
c_{t}^{N}\left(M_{t}^{N}\left(z_{t}^{N}\right)-M_{t}\left(z_{t}^{N}\right)\right)=c_{0}^{N}\left(M_{0}^{N}\left(z_{0}^{N}\right)-M_{0}\left(z_{0}^{N}\right)\right)+m_{t}^{N}\left(z^{N}, c^{N}\right) .
$$

Let $b_{s}^{N}=\Im\left(z_{s}^{N}\right)$, noting that $\left|b_{t}^{N}\right| \geqslant|b|$ for $t \in[0, \tau]$. Let $v_{s}^{N}=\left|c_{s}^{N}\right|^{2}$ and $a_{s}^{N}=$ $\Re\left(\partial_{z} M_{s}^{N}\left(z_{s}^{N}\right)+\partial_{z} M_{s}\left(z_{s}^{N}\right)\right)$, noting that

$$
\left|a_{s}^{N}\right| \leqslant\left|\partial_{z} M_{s}^{N}\left(z_{s}^{N}\right)\right|+\left|\partial_{z} M_{s}\left(z_{s}^{N}\right)\right| \leqslant \frac{2}{\left|b_{s}^{N}\right|^{2}} \leqslant \frac{2}{|b|^{2}},
$$

whereas $\partial_{t} v_{t}^{N}=a_{t}^{N} v_{t}^{N}, v_{\tau}^{N}=1$, by (3.5). Since $v_{t}^{N}=\exp \left(-\int_{t}^{\tau} a_{s}^{N} d s\right)$, it follows that

$$
\sup _{t \in[0, \tau]}\left|c_{t}^{N}\right| \leqslant \exp \left(|b|^{-2}\right) .
$$

Thus, by (3.2), for any $t \in[0, \tau]$,

$$
\begin{aligned}
& \left\langle\Re\left(m^{N}\left(z^{N}, c_{.}^{N}\right)\right)\right\rangle_{t}+\left\langle\Im\left(m^{N}\left(z^{N}, c_{.}^{N}\right)\right)\right\rangle_{t} \\
& =\frac{1}{N^{2}} \int_{0}^{t} \int\left|\frac{c_{s}^{N}}{\left(z_{s}^{N}-x\right)^{2}}\right|^{2} d \hat{\mu}_{s}^{N}(x) d s \leqslant \frac{\mathrm{e}^{2|b|^{-2}}}{N^{2}|b|^{4}}
\end{aligned}
$$

implying that

$$
\mathbb{E}\left|m_{t}^{N}\left(z^{N}, c^{N}\right)\right|^{2}=\mathbb{E}\left\langle\Re\left(m^{N}\left(z^{N}, c^{N}\right)\right)\right\rangle_{t}+\mathbb{E}\left\langle\Im\left(m^{N}\left(z^{N}, c^{N}\right)\right)\right\rangle_{t} \leqslant \frac{\mathrm{e}^{2|b|^{-2}}}{N^{2}|b|^{4}} .
$$

We have by (3.10) and assumption (A) that

$$
\left|c_{0}^{N}\left(M_{0}^{N}\left(z_{0}^{N}\right)-M_{0}\left(z_{0}^{N}\right)\right)\right|=\left|c_{0}^{N}\right|\left|\operatorname{tr}_{N}\left(z_{0}^{N}-D_{N}\right)^{-1}-\int\left(z_{0}^{N}-x\right)^{-1} d \mu_{D}(x)\right|
$$




$$
\leqslant \frac{\mathrm{e}^{|b|^{-2}} \tilde{c}(|b|)}{N}
$$

With $c_{\tau}^{N}=1$, we thus see that (3.8) yields the bound

$$
\mathbb{E}\left[\left|M_{\tau}^{N}\left(z_{\tau}^{N}\right)-M_{\tau}\left(z_{\tau}^{N}\right)\right|^{2}\right]^{1 / 2} \leqslant \frac{\mathrm{e}^{|b|^{-2}} \tilde{c}(|b|)}{N}+\frac{\mathrm{e}^{|b|^{-2}}}{N|b|^{2}} .
$$

Since $z \in \mathbb{C} \backslash \mathbb{R}, N$ and $\tau \in[0,1]$ are arbitrary, this completes the proof of (3.1) when $j=1$, in the Hermitian case. Still in the Hermitian case, let us now prove it for $j=2$ and, without loss of generality, for $z \in \mathbb{C}_{+} \backslash \mathbb{R}$. First, observe that

$$
\begin{aligned}
& \left|\operatorname{tr}_{N}\left(z-X_{N}(\tau)\right)^{-2}+N\left(M_{\tau}^{N}\left(z+\frac{1}{N}\right)-M_{\tau}^{N}(z)\right)\right| \leqslant \frac{1}{N|b|^{3}}, \\
& \left|\int(z-x)^{-2} d \mu_{\tau}^{*}(x)+N\left(M_{\tau}\left(z+\frac{1}{N}\right)-M_{\tau}(z)\right)\right| \leqslant \frac{1}{N|b|^{3}} .
\end{aligned}
$$

Therefore, it is enough to bound

$$
\eta_{\tau}^{N}:=N\left(M_{\tau}^{N}\left(z+\frac{1}{N}\right)-M_{\tau}^{N}(z)\right)-N\left(M_{\tau}\left(z+\frac{1}{N}\right)-M_{\tau}(z)\right)
$$

We proceed as above by considering a martingale representation of $\eta_{\tau}^{N}$, given, if $\left(z_{t}^{N}(z), c_{t}^{N}(z)\right)$ are the functions constructed in (3.4) and (3.5) with terminal data $\left(z_{\tau}^{N}(z), c_{\tau}^{N}(z)\right)=(z, 1)$, by

$$
\begin{aligned}
\eta_{t}^{N}= & N c_{t}^{N}\left(z+\frac{1}{N}\right)\left(M_{t}^{N}\left(z_{t}^{N}\left(z+\frac{1}{N}\right)\right)-M_{t}\left(z_{t}^{N}\left(z+\frac{1}{N}\right)\right)\right) \\
& -N c_{t}^{N}(z)\left(M_{t}^{N}\left(z_{t}^{N}(z)\right)-M_{t}\left(z_{t}^{N}(z)\right)\right) .
\end{aligned}
$$

By (3.8),

$\eta_{t}^{N}=\eta_{0}^{N}+N\left(m_{t}^{N}\left(z_{.}^{N}\left(z+\frac{1}{N}\right), c_{.}^{N}\left(z+\frac{1}{N}\right)\right)-\left(m_{t}^{N} z^{N}(z), c^{N}(z)\right)\right):=\eta_{0}^{N}+\bar{m}_{t}^{N}(z)$.

Note that since $\Im\left(z_{t}^{N}\left(z^{\prime}\right)\right) \geqslant \Im(z)=b>0$ for $z^{\prime}=z$ or $z^{\prime}=z+N^{-1}$, (3.3) and (3.4) imply that

$$
\left|\partial_{t}\left(z_{t}^{N}\left(z+\frac{1}{N}\right)-z_{t}^{N}(z)\right)\right| \leqslant|b|^{-2}\left|z_{t}^{N}\left(z+\frac{1}{N}\right)-z_{t}^{N}(z)\right|,
$$

whereas (3.5), (3.9) and (3.10) imply that

$$
\begin{aligned}
& \left|\partial_{t}\left(c_{t}^{N}\left(z+\frac{1}{N}\right)-c_{t}^{N}(z)\right)\right| \\
& \quad \leqslant \mathrm{e}^{|b|^{-2}}|b|^{-3}\left|z_{t}^{N}\left(z+\frac{1}{N}\right)-z_{t}^{N}(z)\right|+|b|^{-2}\left|c_{t}^{N}\left(z+\frac{1}{N}\right)-c_{t}^{N}(z)\right| .
\end{aligned}
$$

Therefore, Gronwall's lemma gives for $t \leqslant \tau$ 


$$
\begin{aligned}
& \left|z_{t}^{N}\left(z+\frac{1}{N}\right)-z_{t}^{N}(z)\right| \leqslant \frac{1}{N} \mathrm{e}^{(\tau-t) /|b|^{2}}, \\
& \left|c_{t}^{N}\left(z+\frac{1}{N}\right)-c_{t}^{N}(z)\right| \leqslant \frac{1}{N|b|^{3}} \mathrm{e}^{(2(\tau-t)+1) /|b|^{2}} .
\end{aligned}
$$

Using the above control, we can bound $\eta_{0}^{N}$. To this end, let

$$
\begin{aligned}
\eta_{0}^{N}= & N c_{0}^{N}\left(z+\frac{1}{N}\right)\left\{M_{0}^{N}\left(z_{0}^{N}\left(z+\frac{1}{N}\right)\right)-M_{0}^{N}\left(z_{0}^{N}(z)\right)-\left(M_{0}\left(z_{0}^{N}\left(z+\frac{1}{N}\right)\right)\right.\right. \\
& \left.\left.-M_{0}\left(z_{0}^{N}(z)\right)\right)\right\}+N\left(c_{0}^{N}\left(z+\frac{1}{N}\right)-c_{0}^{N}(z)\right)\left\{M_{0}^{N}\left(z_{0}^{N}(z)\right)-M_{0}\left(z_{0}^{N}(z)\right)\right\} \\
= & I+I I .
\end{aligned}
$$

The first term can be decomposed as follows

$$
\begin{aligned}
I= & -N c_{0}^{N}\left(z+\frac{1}{N}\right)\left(z_{0}^{N}\left(z+\frac{1}{N}\right)-z_{0}^{N}(z)\right) \\
& \times\left\{\operatorname{tr}_{N}\left(z_{0}^{N}(z)-D_{N}\right)^{-2}-\int\left(z_{0}^{N}(z)-x\right)^{-2} d \mu_{D}(x)\right\} \\
& +N c_{0}^{N}\left(z+\frac{1}{N}\right)\left(z_{0}^{N}\left(z+\frac{1}{N}\right)-z_{0}^{N}(z)\right)^{2} \\
& \times\left\{\int\left(z_{0}^{N}\left(z+\frac{1}{N}\right)-x\right)^{-1}\left(z_{0}^{N}(z)-x\right)^{-2} d\left(\hat{\mu}_{0}^{N}-\mu_{D}\right)(x)\right\}=I_{1}+I_{2} .
\end{aligned}
$$

By (3.10), (3.14) and assumption (A) we find that

$$
\left|I_{1}\right| \leqslant N \mathrm{e}^{|b|^{-2}} \frac{1}{N} \mathrm{e}^{\tau /|b|^{2}} \frac{\tilde{c}(|b|)}{N}
$$

and

$$
\left|I_{2}\right| \leqslant N \mathrm{e}^{|b|^{-2}} \frac{\mathrm{e}^{2 \tau /|b|^{2}}}{N^{2}} \frac{1}{|b|^{3}}
$$

so that we have found a finite constant $C_{1}(b)$ such that

$$
|I| \leqslant \frac{C_{1}(b)}{N} .
$$

Moreover, by (3.14) and assumption (A),

$$
|I I| \leqslant N \frac{1}{N|b|^{3}} \mathrm{e}^{(2 \tau+1) /|b|^{2}} \frac{\tilde{c}(|b|)}{N}
$$

resulting, with (3.15), with the existence of a finite constant $C_{2}(b)$ such that

$$
\left|\eta_{0}^{N}\right| \leqslant \frac{C_{2}(b)}{N} .
$$


Moreover $\bar{m}^{N}(z)$ is a martingale whose martingale bracket can be computed. Following (3.11), we find

$$
\begin{aligned}
& \left\langle\Re\left(\bar{m}^{N}(z)\right)\right\rangle_{\tau}+\left\langle\Im\left(\bar{m}^{N}(z)\right)\right\rangle_{\tau} \\
& =\frac{1}{N^{2}} \int_{0}^{\tau} \int\left|\frac{N c_{s}^{N}\left(z+N^{-1}\right)}{\left(z_{s}^{N}\left(z+N^{-1}\right)-x\right)^{2}}-\frac{N c_{s}^{N}(z)}{\left(z_{s}^{N}(z)-x\right)^{2}}\right|^{2} d \hat{\mu}_{s}^{N}(x) d s \\
& \leqslant \frac{1}{N^{2}} \int_{0}^{\tau}\left(\frac{\left(N\left|c_{s}^{N}\left(z+N^{-1}\right)-c_{s}^{N}(z)\right|\right)^{2}}{\left|b_{s}^{N}\left(z+N^{-1}\right)\right|^{4}}\right) d s \\
& \quad+\frac{1}{N^{2}} \int_{0}^{\tau}\left(\frac{\left|c_{s}^{N}(z)\right|^{2}\left|N\left(z_{s}^{N}\left(z+N^{-1}\right)-z_{s}^{N}(z)\right)\right|^{2}}{\left(\left|b_{s}^{N}(z)\right| \wedge\left|b_{s}^{N}\left(z+N^{-1}\right)\right|\right)^{6}}\right) d s \\
& \leqslant \frac{C_{3}(b)}{N^{2}}
\end{aligned}
$$

where $C_{3}(b)$ is a finite constant derived from (3.10) and (3.14). From (3.12), (3.13), (3.16) and (3.17), we conclude that

$$
\begin{aligned}
& \mathbb{E}\left[\left|\operatorname{tr}_{N}\left(z-X_{N}(\tau)\right)^{-2}-\int(z-x)^{-2} d \mu^{*}(x)\right|^{2}\right]^{1 / 2} \\
& \leqslant \frac{2}{N|b|^{3}}+\frac{C_{2}(b)}{N}+\frac{C_{3}(b)^{1 / 2}}{N}=\frac{C_{4}(b)}{N}
\end{aligned}
$$

finishing the proof of the lemma in the Hermitian case. When we consider the symmetric case (studied already by [13]), an extra term of the form $(2 N)^{-1} \int_{0}^{\tau} c_{s}^{N} \partial_{z}^{2} M_{s}^{N}\left(z_{s}^{N}\right) d s$ appears in (3.8). This term is in turn bounded by $C N^{-1} \log \left(1+\frac{1}{|b|}\right)$ (see [13, p. 9] for details), completing the proof of the lemma.

\section{A martingale representation for $M^{N}(z)$}

In Section 3, we used the martingale representation (3.8) of $M_{.^{N}}\left(z^{N}\right)$ to estimate its rate of convergence as $N \rightarrow \infty$. Here, we shall follow more closely [9] and [5] to get a similar representation but for deterministic functions $\left(c_{t}, z_{t}\right)$, independent of $N$, in order to study the moderate deviations of the sequence $\left\{M^{N}(z)-M(z)\right\}_{N}$. To this end, let $(c ., z$.) be the solution of

$$
\begin{aligned}
\partial_{t} z_{t} & =M_{t}\left(z_{t}\right), \quad z_{1}=z=a+\mathrm{i} b, \\
\partial_{t} c_{t} & =\partial_{z} M_{t}\left(z_{t}\right) c_{t}, \quad c_{1}=c .
\end{aligned}
$$

By the same arguments as above, we see that $\left|\Im\left(z_{t}\right)\right|$ is non-increasing on $[0,1]$, with existence and uniqueness of $\left(c_{t}, z_{t}\right)$ as a result. Further, in analogy with (3.8) one finds that

$$
c\left(M_{1}^{N}\left(z_{1}\right)-M_{1}\left(z_{1}\right)\right)=c_{0}\left(M_{0}^{N}\left(z_{0}\right)-M_{0}\left(z_{0}\right)\right)+r_{1}^{N}(z ., c .)+m_{1}^{N}(z ., c .)
$$

with $m_{1}^{N}(z ., c$.$) the martingale of (3.2) and$ 


$$
\begin{aligned}
r_{1}^{N}(z ., c .)= & -\int_{0}^{1} c_{t}\left(M_{t}^{N}\left(z_{t}\right)-M\left(z_{t}\right)\right) \partial_{z}\left(M_{t}^{N}\left(z_{t}\right)-M\left(z_{t}\right)\right) d t \\
& +\mathbf{1}_{\beta=1}(2 N)^{-1} \int_{0}^{1} c_{t} \partial_{z}^{2} M_{t}^{N}\left(z_{t}\right) d t .
\end{aligned}
$$

We claim that

LEMmA 4.1. - For any $z \in \mathbb{C} \backslash \mathbb{R}, c \in \mathbb{C}$ and $a_{N} \rightarrow 0$ such that $N a_{N} \rightarrow \infty$,

$$
\limsup _{N \rightarrow \infty}\left(N a_{N}\right)^{-2} \log \mathbb{P}\left(\left|r_{1}^{N}(z, c .)\right| \geqslant a_{N}\right)=-\infty .
$$

Proof. - Let $b_{t}=\Im\left(z_{t}\right)$. From (4.1) we see that $\left|\partial_{t} b_{t}\right| \leqslant\left|\partial_{t} z_{t}\right| \leqslant 1 /\left|b_{t}\right| \leqslant 1 /|b|$. So, with $b_{1}=b$, we have that $\sup _{t \in[0,1]}\left|b_{t}\right|<\infty$. In analogy with the derivation of (3.10), we have by (4.1) and (4.2) that

$$
C_{1}=C_{1}(b, c):=\sup _{t \in[0,1]}\left|c_{t}\right|<\infty .
$$

Notice that

$$
\mathbf{1}_{\beta=1}(2 N)^{-1}\left|\int_{0}^{1} c_{s} \partial_{z}^{2} M_{s}^{N}\left(z_{s}\right) d s\right| \leqslant N^{-1} C_{1}|b|^{-3},
$$

so without loss of generality we may and shall ignore this term, considering hereafter $\beta=2$. Recall [11], that if $f$ is Lipschitz of norm $\|f\|_{\mathrm{L}}:=\sup _{x \neq y}(|f(x)-f(y)| / \mid x-$ $y \mid)$, then

$$
\left(\omega_{i j}(t)\right)_{1 \leqslant i, j \leqslant N}:=\left(\beta_{i j}(t), \tilde{\beta}_{i j}(t)\right)_{1 \leqslant i \leqslant j \leqslant N} \mapsto\left(\operatorname{tr}_{N} f\left(X_{N}(t)\right)-\mathbb{E} \operatorname{tr}_{N} f\left(X_{N}(t)\right)\right)
$$

is Lipschitz for the Euclidean norm with constant $2\|f\|_{\mathrm{L}} / N$ (this was shown in [11] for $D_{N}=0$, but the proof of [11, Lemma 1.2(b)] extends verbatim to the general case, hence all conclusions of [11] extend as well). Considering $f(x)=(z-x)^{-1}$ and

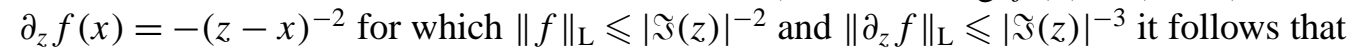
for any $\xi \in \mathbb{C} \backslash \mathbb{R} \times[-|b|,|b|]$, and $t \in[0,1]$,

$\left(\omega_{i j}(t)\right)_{1 \leqslant i, j \leqslant N} \mapsto\left(M_{t}^{N}(\xi)-M_{t}(\xi)\right), \quad$ and $\quad\left(\omega_{i j}(t)\right)_{1 \leqslant i, j \leqslant N} \mapsto \partial_{z}\left(M_{t}^{N}(\xi)-M_{t}(\xi)\right)$

are Lipschitz functions of norm at most $2 /\left(|b|^{2} N\right)$ and $2 /\left(|b|^{3} N\right)$, respectively. Therefore, [11] provides the existence of a universal constant $c>0$ such that for any $\delta>0, N$ and $t \in[0,1]$,

$$
\begin{aligned}
& \mathbb{P}\left(\left|M_{t}^{N}\left(z_{t}\right)-M_{t}\left(z_{t}\right)-\mathbb{E}\left[\left(M_{t}^{N}\left(z_{t}\right)-M_{t}\left(z_{t}\right)\right)\right]\right| \geqslant \delta\right) \leqslant \mathrm{e}^{-c \delta^{2} b^{4} N^{2},} \\
& \mathbb{P}\left(\left|\partial_{z}\left(M_{t}^{N}\left(z_{t}\right)-M_{t}\left(z_{t}\right)\right)-\mathbb{E}\left[\partial_{z}\left(M_{t}^{N}\left(z_{t}\right)-M_{t}\left(z_{t}\right)\right)\right]\right| \geqslant \delta\right) \leqslant \mathrm{e}^{-c \delta^{2} b^{6} N^{2}} .
\end{aligned}
$$

By Lemma 3.1, we have that for some finite $C_{2}(|b|)$ and all $N$,

$$
\sup _{t \in[0,1]}\left|\mathbb{E}\left[\left(M_{t}^{N}\left(z_{t}\right)-M_{t}\left(z_{t}\right)\right)\right]\right| \leqslant C_{2}(|b|) N^{-1}
$$


and

$$
\sup _{t \in[0,1]} \sup _{|\Im(z)| \geqslant|b|} \mathbb{E}\left[\left|\partial_{z}\left(M_{t}^{N}(z)-M_{t}(z)\right)\right|^{2}\right]^{1 / 2} \leqslant C_{2}(|b|) N^{-1} .
$$

Fixing $a_{N} \rightarrow 0$ such that $N a_{N} \rightarrow \infty$, if we let

$$
\psi_{N}:\left(z, \omega_{i j}(t), s\right)_{1 \leqslant i, j \leqslant N} \mapsto\left(M_{t}^{N}(z)-M_{s}(z)\right) \partial_{z}\left(M_{t}^{N}(z)-M_{s}(z)\right),
$$

it follows from (4.5), (4.6), (4.7) and (4.8) that for $K>K_{0}:=C_{2}(|b|) / \inf _{N}\left(N a_{N}\right)$,

$$
\begin{aligned}
& \mathbb{P}\left(\left|\psi_{N}\left(z_{t}, \omega(t), t\right)\right| \geqslant 4 K^{2} a_{N}^{2}\right) \\
& \quad \leqslant \mathbb{P}\left(\left|M_{t}^{N}\left(z_{t}\right)-M_{t}\left(z_{t}\right)\right| \geqslant 2 K a_{N}\right)+\mathbb{P}\left(\left|\partial_{z}\left(M_{t}^{N}\left(z_{t}\right)-M_{t}\left(z_{t}\right)\right)\right| \geqslant 2 K a_{N}\right) \\
& \quad \leqslant 2 \mathrm{e}^{-c K^{2} b^{4}\left(1 \wedge b^{2}\right)\left(a_{N} N\right)^{2}} .
\end{aligned}
$$

Turning to estimate the integral appearing in $r_{t}^{N}(z, c$.$) , note that \left|M_{t}^{N}(z)-M_{s}(z)\right| \leqslant$ $2 /|\Im(z)|,\left|\partial_{z}\left(M_{t}^{N}(z)-M_{s}(z)\right)\right| \leqslant 2 /|\Im(z)|^{2}$, whereas $\omega(t) \mapsto\left(M_{t}^{N}(z)-M_{s}(z)\right)$ and $\omega(t) \mapsto \partial_{z}\left(M_{t}^{N}(z)-M_{s}(z)\right)$ are Lipschitz for the Euclidean norm with constants bounded by $2 /\left(|\Im(z)|^{2} N\right)$ and $2 /\left(|\Im(z)|^{3} N\right)$, respectively. Hence,

$$
\left|\psi_{N}(z, \omega(t), s)-\psi_{N}(z, \omega(s), s)\right| \leqslant \frac{8}{|\Im(z)|^{4} N}\|\omega(t)-\omega(s)\|_{2}
$$

(where $\|\omega\|_{2}^{2}:=\sum_{1 \leqslant i, j \leqslant N} \omega_{i j}^{2}$ ). Moreover,

$$
\begin{aligned}
\left|M_{t}(z)-M_{s}(z)\right| & =\lim _{N \rightarrow \infty}\left|\mathbb{E} \operatorname{tr}_{N}\left[\left(z-X_{N}(t)\right)^{-1}\right]-\mathbb{E} \operatorname{tr}_{N}\left[\left(z-X_{N}(s)\right)^{-1}\right]\right| \\
& =\lim _{N \rightarrow \infty}\left|\mathbb{E} \operatorname{tr}_{N}\left[\left(z-X_{N}(t)\right)^{-1}\left(H_{N}(t)-H_{N}(s)\right)\left(z-X_{N}(s)\right)^{-1}\right]\right| \\
& \leqslant \frac{1}{|\Im(z)|^{2}} \lim _{N \rightarrow \infty} \mathbb{E} \operatorname{tr}_{N}\left[\left|H_{N}(t)-H_{N}(s)\right|\right] \\
& \leqslant \frac{1}{|\Im(z)|^{2}} \lim _{N \rightarrow \infty} \sqrt{\mathbb{E} \operatorname{tr}_{N}\left[\left|H_{N}(t)-H_{N}(s)\right|^{2}\right]}=\frac{|t-s|^{1 / 2}}{|\Im(z)|^{2}}
\end{aligned}
$$

and similarly,

$$
\left|\partial_{z}\left(M_{t}(z)-M_{s}(z)\right)\right| \leqslant \frac{2|t-s|^{1 / 2}}{|\Im(z)|^{3}}
$$

implying that

$$
\begin{aligned}
& \left|\psi_{N}(z, \omega(t), t)-\psi_{N}(z, \omega(t), s)\right| \\
& \quad \leqslant \frac{2}{|\mathfrak{I}(z)|^{2}}\left|M_{t}(z)-M_{s}(z)\right|+\frac{2}{|\mathfrak{I}(z)|}\left|\partial_{z}\left(M_{t}(z)-M_{s}(z)\right)\right| \\
& \quad \leqslant \frac{6|t-s|^{1 / 2}}{|\mathfrak{I}(z)|^{4}} .
\end{aligned}
$$

In view of (3.3) and the analogous bound

$$
\left|\partial_{z} M_{t}^{N}(z)-\partial_{z} M_{t}^{N}(\tilde{z})\right| \vee\left|\partial_{z} M_{s}(z)-\partial_{z} M_{s}(\tilde{z})\right| \leqslant|b|^{-3}|z-\tilde{z}|,
$$


for $|\Im(z)| \wedge|\Im(\tilde{z})| \geqslant|b|$, it follows that

$$
\left|\psi_{N}\left(z_{t}, \omega(t), s\right)-\psi_{N}\left(z_{s}, \omega(t), s\right)\right| \leqslant 8|b|^{-4}\left|z_{t}-z_{s}\right| \leqslant 8|b|^{-5}|t-s|
$$

(the last inequality comes from (4.1), as $\left|\partial_{t} z_{t}\right| \leqslant 1 /|b|$ ). With $n=A a_{N}^{-2}$, we have by (4.4), (4.11), (4.13), and (4.14) that for $N$ large enough,

$$
\begin{aligned}
\left|\int_{0}^{1} c_{s} \psi_{N}\left(z_{s}, \omega(s), s\right) d s\right| \leqslant & C_{1}\left[\frac{a_{N}}{3}+\max _{i=0}^{n-1} \sup _{s \in[i / n,(i+1) / n]}\left|\psi_{N}\left(z_{i / n}, \omega(s), \frac{i}{n}\right)\right|\right] \\
\leqslant & C_{1}\left[\frac{a_{N}}{3}+\max _{i=0}^{n-1}\left|\psi_{N}\left(z_{i / n}, \omega\left(\frac{i}{n}\right), \frac{i}{n}\right)\right|\right. \\
& \left.+8|b|^{-4} N^{-1} \max _{i=0}^{n-1} \sup _{s \in[i / n,(i+1) / n]}\left\|\omega(s)-\omega\left(\frac{i}{n}\right)\right\|_{2}\right] .
\end{aligned}
$$

So, by (4.10) with $K=K_{N}=\left(12 a_{N}\right)^{-1 / 2}$, for some $C_{4}=C_{4}(b)>0$,

$$
\begin{aligned}
& \mathbb{P}\left(\left|\int_{0}^{1} c_{s} \psi_{N}\left(z_{s}, \omega(s), s\right) d s\right| \geqslant C_{1} a_{N}\right) \\
& \quad \leqslant 2 n \mathrm{e}^{-C_{4} a_{N} N^{2}}+n \mathbb{P}\left(\sup _{s \in[0,1 / n]}\|\omega(s)\|_{2} \geqslant \frac{|b| a_{N} N}{24}\right) .
\end{aligned}
$$

Combining Brownian scaling, Chebyshev's inequality and Désiré André reflection principle, we see that for any $C=C_{5}(b)$,

$$
\begin{aligned}
n \mathbb{P}\left(\sup _{s \in[0,1 / n]}\|\omega(s)\|_{2}^{2} \geqslant C\left(a_{N} N\right)^{2}\right) & \leqslant n \mathbb{P}\left(\sum_{i, j=1}^{N} \sup _{\theta \in[0,1]} \omega_{i j}(\theta)^{2} \geqslant A C N^{2}\right) \\
& \leqslant n\left\{2 \mathrm{e}^{-A C / 3} \mathbb{E}\left[\mathrm{e}^{\omega_{11}(1)^{2} / 3}\right]\right\}^{N^{2}} \leqslant \mathrm{e}^{-N^{2}},
\end{aligned}
$$

provided $A \geqslant A_{0}(b)$ is large enough. Thus, with $N a_{N} \rightarrow \infty$ and $a_{N} \rightarrow 0$, it follows from (4.15) that

$$
\limsup _{N \rightarrow \infty}\left(N a_{N}\right)^{-2} \log \mathbb{P}\left(\left|r_{1}^{N}(z, c .)\right| \geqslant C_{1} a_{N}\right) \leqslant \limsup _{N \rightarrow \infty} a_{N}^{-1}\left[2 a_{N}\left|\log a_{N}\right|-C_{4}\right]=-\infty,
$$

as needed to complete the proof.

\section{Moderate deviations}

For $d<\infty$ and $\mathbf{f}=\left(f^{(1)}, \ldots, f^{(d)}\right), f^{(i)} \in \mathcal{S}$ tieljes $(\mathbb{R})$, let

$$
X_{N}(\mathbf{f}):=\left(\int \mathbf{f}(x) d \hat{\mu}_{1}^{N}(x)-\int \mathbf{f}(x) d \mu^{*}(x)\right) \in \mathbb{R}^{d} .
$$

We next prove that $X_{N}(\mathbf{f})$ satisfies the moderate deviation principle in $\mathbb{R}^{d}$ per fixed $\mathbf{f} \in \mathcal{S} \operatorname{tieljes}(\mathbb{R})^{d}$. 
THEOREM 5.1. - For any $a_{N} \rightarrow 0$ such that $N a_{N} \rightarrow \infty$, any $d<\infty$ and $\mathbf{f} \in$ $(\mathcal{S} \text { tieljes }(\mathbb{R}))^{d}$, the sequence of random vectors $\left\{a_{N}^{-1} X_{N}(\mathbf{f})\right\}_{N}$ satisfies the LDP in $\mathbb{R}^{d}$, with speed $\left(N a_{N}\right)^{-2}$ and the good rate function

$$
I_{\mathbf{f}}(\boldsymbol{x})=\frac{\beta}{2} \sup _{\lambda_{1}, \ldots, \lambda_{d}}\left\{\sum_{i=1}^{d} \lambda_{i} x_{i}-\frac{1}{2} \sum_{i, j=1}^{d} \lambda_{i} \lambda_{j} \mathcal{V}_{1}\left(f^{(i)}, f^{(j)}\right)\right\},
$$

for $\mathcal{V}_{1}(f, g)$ of (1.2).

In particular, considering $d=1$ and $\beta=2$, we see that the good rate function for the LDP of $a_{N}^{-1} X_{N}(h)$ in $\mathbb{R}$ is $x^{2} /\left(2 \mathcal{V}_{1}(h, h)\right)$. Since $N a_{N} \rightarrow \infty$ and $h \in \mathcal{S}$ tieljes $(\mathbb{R})$ is a Lipschitz function, it follows from [11, Theorem 1.1(b)], after some algebra, that

$$
\limsup _{N \rightarrow \infty}\left(N a_{N}\right)^{-2} \log \mathbb{E}\left(\mathrm{e}^{\lambda N^{2} a_{N} \bar{X}_{N}(h)}\right)<\infty,
$$

for all $\lambda<\infty$. From Lemma 3.1 we see that the same applies when $\bar{X}_{N}(h)$ is replaced by $X_{N}(h)$, and (1.5) then follows by applying Varadhan's lemma (see, for example, [8, Theorem 4.5.10]).

Proof. - Our strategy consists of applying theorem [17, Theorem 2.2] that yields the moderate deviations principle for martingales. Some preparations are needed in order to check that its conditions are satisfied in our setup. We first consider the Hermitian case $\beta=2$.

Any $h \in \mathcal{S}$ tieljes $(\mathbb{R})$ is of the form

$$
h(x):=\sum_{k=1}^{\ell} c^{(k)}\left(z^{(k)}-x\right)^{-1},
$$

for some $\ell<\infty, c^{(k)} \in \mathbb{C}$ and $z^{(k)}=a^{(k)}+\mathrm{i} b^{(k)}$ with $b^{(k)} \neq 0$. Combining assumption (A) with (4.4) we have that

$$
\left|c_{0}^{(k)}\left(M_{0}^{N}\left(z_{0}^{(k)}\right)-M_{0}\left(z_{0}^{(k)}\right)\right)\right| \leqslant \frac{C^{(k)}}{N}
$$

for some $C^{(k)}<\infty$ and all $N$. Applying Lemma 4.1 and the representation (4.3) for $k=$ $1, \ldots, \ell$, it thus follows that $\left\{a_{N}^{-1} m_{1}^{N}(h)\right\}_{N}$ is exponentially equivalent to $\left\{a_{N}^{-1} X_{N}(h)\right\}_{N}$ at speed $\left(N a_{N}\right)^{-2} \rightarrow 0$, in the sense of [8, Definition 4.2.10], where,

$$
m_{t}^{N}(h):=\sum_{k=1}^{\ell} m_{t}^{N}\left(z^{(k)}, c^{(k)}\right),
$$

is the continuous martingale $S^{0, t}\left(\hat{\mu}^{N}, h\right)$ (or $\widetilde{S}^{0, t}\left(\hat{\mu}^{N}, h\right)$ ), of Theorem 2.1. Hence, by [8, Theorem 4.2.13], it suffices to prove the LDP at speed $\left(N a_{N}\right)^{-2}$ and good rate function $I_{\mathbf{f}}(\boldsymbol{x})$ for

$$
a_{N}^{-1} m_{1}^{N}(\mathbf{f}):=a_{N}^{-1}\left(m_{1}^{N}\left(f^{(1)}\right), \ldots, m_{1}^{N}\left(f^{(d)}\right)\right) \in \mathbb{R}^{d} .
$$


To this end, note that the continuous martingale $a_{N}^{-1} m_{t}^{N}(\mathbf{f})$, with $a_{N}^{-1} m_{0}^{N}(\mathbf{f})=\mathbf{0}$, trivially satisfies Cramér's condition [17, (2.6)] on the compensator, while the differentiable everywhere function (in $\lambda_{1}, \ldots, \lambda_{d}$ ),

$$
G_{t}(\lambda):=\frac{1}{2} \sum_{i, j=1}^{d} \lambda_{i} \lambda_{j} \mathcal{V}_{t}\left(f^{(i)}, f^{(j)}\right)
$$

satisfies the strict convexity condition $[17,(\mathrm{G})]$ (see the discussion in $[17, \mathrm{p} .49])$. Hence, with $n=\left(N a_{N}\right)^{2}$, by [17, Theorem 2.2] the sequence $\left\{a_{N}^{-1} m^{N}(\mathbf{f})\right\}$ satisfies the LDP of speed $\left(N a_{N}\right)^{-2}$ in $\mathcal{D}[0,1]$, equipped with the Skorohod topology, provided that for any $\delta>0$,

$$
\limsup _{N \rightarrow \infty} N^{-2} \log \mathbb{P}\left(\sup _{t \in[0,1]}\left|N^{2}\left\langle m^{N}(h)\right\rangle_{t}-\mathcal{V}_{t}(h, h)\right|>2 \delta\right)<0,
$$

where $h(x):=\sum_{i=1}^{d} \lambda_{i} f^{(i)}(x) \in \mathcal{S}$ tieljes $(\mathbb{R})$ (after some algebra one sees that this is exactly condition $[17,(\sup \mathcal{E})]$ in our context). Let $\boldsymbol{V}_{t}$ denote the matrix of entries $V_{i j}(t):=\frac{d}{d t} \mathcal{V}_{t}\left(f^{(i)}, f^{(j)}\right)$. For each $\boldsymbol{x} \in \mathbb{R}^{d}$ and positive semi-definite matrix $\boldsymbol{V}=$ $\left\{V_{i j}\right\}_{i, j=1}^{d}$, let

$$
L(\boldsymbol{x}, \boldsymbol{V}):=\sup _{\lambda_{1}, \ldots, \lambda_{d}}\left\{\sum_{i=1}^{d} \lambda_{i} x_{i}-\frac{1}{2} \sum_{i, j=1}^{d} \lambda_{i} \lambda_{j} V_{i j}\right\}
$$

By [17, (2.4)], the good rate function $J_{\mathbf{f}}(\cdot)$ for the $\operatorname{LDP}$ of $\left\{a_{N}^{-1} m^{N}(\mathbf{f})\right\}$ is $\int_{0}^{1} L\left(\dot{\phi}(t), \boldsymbol{V}_{t}\right) d t$, for $\phi(\cdot)$ absolutely continuous with $\phi(0)=0$, and infinite otherwise. Since $a_{N}^{-1} m^{N}(\mathbf{f}) \in$ $\mathcal{C}[0,1]$ and $\left\{\phi: J_{\mathbf{f}}(\phi)<\infty\right\} \subset \mathcal{C}[0,1]$, it follows from [17, Theorem $\left.\mathrm{C}\right]$ that the same LDP applies in $\mathcal{C}[0,1]$ equipped with the uniform topology. The LDP for $\left\{a_{N}^{-1} m_{1}^{N}(\mathbf{f})\right\}$ with the good rate function $I_{\mathbf{f}}(\boldsymbol{x})=L\left(\boldsymbol{x}, \int_{0}^{1} \boldsymbol{V}_{t} d t\right)$ then follows by the contraction principle for $\phi(\cdot) \mapsto \phi(1)$ and the convexity of $(\boldsymbol{x}, \boldsymbol{V}) \mapsto L(\boldsymbol{x}, \boldsymbol{V})$.

We turn to the remaining task of proving that (5.3) holds for any $\delta>0$ and $h \in$ $\mathcal{S}$ tieljes $(\mathbb{R})$. Fixing $\delta$ and $h$, since the monotone function $t \mapsto \mathcal{V}_{t}(h, h)$ is uniformly continuous on $[0,1]$,

$$
\sup _{0 \leqslant s \leqslant t \leqslant 1, t-s \leqslant 1 / n}\left\{\mathcal{V}_{t}(h, h)-\mathcal{V}_{s}(h, h)\right\}<\delta,
$$

for some $n=n(\delta)<\infty$, so with $N^{2}\left\langle m^{N}(h)\right\rangle_{t}$ non-decreasing in $t$, (5.3) follows as soon as we show that

$$
\limsup _{N \rightarrow \infty} N^{-2} \log \mathbb{P}\left(\left|N^{2}\left\langle m^{N}(h)\right\rangle_{t}-\mathcal{V}_{t}(h, h)\right|>\delta\right)<0,
$$

for any fixed $t \in[0,1]$. Recall that

$$
N^{2}\left\langle m^{N}(h)\right\rangle_{t}-\mathcal{V}_{t}(h, h)=\int_{0}^{t}\left[\int\left(\partial_{x} h_{s}\right)^{2}(x) d \hat{\mu}_{s}^{N}(x)-\int\left(\partial_{x} h_{s}\right)^{2}(x) d \mu_{s}^{*}(x)\right] d s .
$$


By the linearity of $h \mapsto h_{s}$ via (1.1), the right-hand side of (5.5) is a quadratic form in $c^{(k)}, k=1, \ldots, \ell$, of the finite decomposition (5.2) for $h \in \mathcal{S}$ tieljes $(\mathbb{R})$. Thus, for proving (5.4), we may and shall replace $\left(\partial_{x} h_{s}\right)^{2}$ in (5.5) by $g\left(z_{s}, \tilde{z}_{s}, x\right):=\left[\left(z_{s}-x\right)\left(\tilde{z}_{s}-\right.\right.$ $x)]^{-2}$, where $z_{s}$ and $\tilde{z}_{s}$ are the solutions of (4.1) for $z_{1}=z$ and $z_{1}=\tilde{z}$, respectively. Doing so, we need only show that for any $\eta>0$ and $z, \tilde{z} \in \mathbb{C} \backslash \mathbb{R}$,

$$
\limsup _{N \rightarrow \infty} N^{-2} \log \mathbb{P}\left(\int_{0}^{1}\left|\phi_{N}\left(z_{s}, \tilde{z}_{s}, \omega(s), s\right)\right| d s>5 \eta\right)<0,
$$

where for $u, v \in \mathbb{C} \backslash \mathbb{R}, s \in[0,1]$ and $W \in \mathcal{M}_{N}$,

$$
\phi_{N}:(u, v, W, s) \mapsto \operatorname{tr}_{N} g(u, v, W)-\int g(u, v, x) d \mu_{s}^{*}(x) .
$$

Let $b=|\Im(z)| \wedge|\Im(\tilde{z})|>0$. Consider the bounded-Lipschitz norm

$$
\|v\|_{B L}:=\sup \left\{\left|\int f d v\right|:\|f\|_{L}+\|f\|_{\infty} \leqslant 1\right\}
$$

on the space of Borel measures on $\mathbb{R}$. Note that $\left\|\hat{\mu}_{t}^{N}-\mu_{t}^{*}\right\|_{B L} \rightarrow 0$ in probability, as $N \rightarrow \infty$, for each $t \in[0,1]$. Since $\left\|g\left(z_{s}, \tilde{z}_{s}, \cdot\right)\right\|_{\infty} \leqslant b^{-4}$ and $\left\|g\left(z_{s}, \tilde{z}_{s}, \cdot\right)\right\|_{\mathrm{L}} \leqslant 4 b^{-5}$, it follows that

$$
\left|\mathbb{E} \operatorname{tr}_{N} g\left(z_{t}, \tilde{z}_{t}, X_{N}(t)\right)-\int g\left(z_{t}, \tilde{z}_{t}, x\right) d \mu_{t}^{*}(x)\right| \leqslant \eta,
$$

for all $N \geqslant N_{0}(\eta, t)$. It also follows by [11, Theorem 1.1b] that for some $c>0$ and all $N, \eta>0, t \in[0,1]$,

$$
\mathbb{P}\left(\left|\operatorname{tr}_{N} g\left(z_{t}, \tilde{z}_{t}, X_{N}(t)\right)-\mathbb{E}\left(\operatorname{tr}_{N} g\left(z_{t}, \tilde{z}_{t}, X_{N}(t)\right)\right)\right| \geqslant \eta\right) \leqslant \mathrm{e}^{-c \eta^{2} b^{10} N^{2}} .
$$

By the same argument leading to (4.12) we see that $\left\|\mu_{t}^{*}-\mu_{s}^{*}\right\|_{B L} \leqslant \sqrt{|t-s|}$. Moreover, since $\left\|g\left(z_{s}, \tilde{z}_{s}, \cdot\right)\right\|_{\mathrm{L}} \leqslant 4 b^{-5}$, and $\left|g\left(z_{s}, \tilde{z}_{s}, x\right)-g\left(z_{t}, \tilde{z}_{t}, x\right)\right| \leqslant 2 b^{-5}\left(\left|\tilde{z}_{t}-\tilde{z}_{s}\right|+\left|z_{t}-z_{s}\right|\right)$, similarly to (4.11) and (4.14) we have that for some $C_{4}=C_{4}(|b|)<\infty$,

$$
\begin{aligned}
&\left|\phi_{N}\left(z_{t}, \tilde{z}_{t}, \omega(t), t\right)-\phi_{N}\left(z_{s}, \tilde{z}_{s}, \omega(s), s\right)\right| \\
& \leqslant \mid\left|\phi_{N}\left(z_{t}, \tilde{z}_{t}, \omega(t), t\right)-\phi_{N}\left(z_{t}, \tilde{z}_{t}, \omega(t), s\right)\right| \\
& \quad+\left|\phi_{N}\left(z_{t}, \tilde{z}_{t}, \omega(t), s\right)-\phi_{N}\left(z_{t}, \tilde{z}_{t}, \omega(s), s\right)\right| \\
& \quad+\left|\phi_{N}\left(z_{t}, \tilde{z}_{t}, \omega(s), s\right)-\phi_{N}\left(z_{t}, \tilde{z}_{s}, \omega(s), s\right)\right| \\
& \quad+\left|\phi_{N}\left(z_{t}, \tilde{z}_{s}, \omega(s), s\right)-\phi_{N}\left(z_{s}, \tilde{z}_{s}, \omega(s), s\right)\right| \\
& \leqslant \frac{b+4}{b^{5}}|t-s|^{1 / 2}+\frac{8}{b^{5} N}\|\omega(t)-\omega(s)\|_{2}+\frac{4}{b^{5}}\left|\tilde{z}_{t}-\tilde{z}_{s}\right|+\frac{4}{b^{5}}\left|z_{t}-z_{s}\right| \\
& \leqslant C_{4}\left(\frac{1}{N}\|\omega(t)-\omega(s)\|_{2}+|t-s|^{1 / 2}\right) .
\end{aligned}
$$

Taking $n=A / \eta^{2}$, we then have for all $A>A_{0}(b)$, 


$$
\begin{aligned}
\int_{0}^{1}\left|\phi_{N}\left(z_{s}, \tilde{z}_{s}, \omega(s), s\right)\right| d s \leqslant & +\max _{i=0}^{n-1}\left|\phi_{N}\left(z_{i / n}, \tilde{z}_{i / n}, \omega\left(\frac{i}{n}\right), \frac{i}{n}\right)\right| \\
& +\frac{8}{b^{5} N} \max _{i=0}^{n-1} \sup _{s \in[i / n,(i+1) / n]}\left\|\omega(s)-\omega\left(\frac{i}{n}\right)\right\|_{2} .
\end{aligned}
$$

We now have (5.6) by combining the latter inequality, (5.7), (5.8) applied at $t=i / n, i=$ $0, \ldots, n-1$, and the fact that

$$
n \mathbb{P}\left(\sup _{s \in[0,1 / n]}\|\omega(s)\|_{2}^{2} \geqslant C \eta^{2} N^{2}\right) \leqslant n \mathbb{P}\left(\sum_{i, j=1}^{N} \sup _{\theta \in[0,1]} \omega_{i j}(\theta)^{2} \geqslant A C N^{2}\right) \leqslant \mathrm{e}^{-N^{2}},
$$

which holds for all $A$ large enough. For symmetric matrices, note that the only difference is that the bracket of the martingales $m^{N}(h)$ is twice what it is in the Hermitian case (see Theorem 2.1).

Proof of Theorem 1.1. - Equip the algebraic dual $\mathcal{X}$ of $\mathcal{S}$ tieljes' $(\mathbb{R})$ with the $\mathcal{S}$ tieljes' $(\mathbb{R})$-topology and the smallest $\sigma$-field $\mathcal{A}$ such that $F \mapsto\left\langle f^{\prime}, F\right\rangle: \mathcal{X} \rightarrow \mathcal{B}_{\mathbb{R}}$ are measurable for each $f^{\prime} \in \mathcal{S}$ tieljes $^{\prime}(\mathbb{R})$. We note that $\mathcal{S}$ tieljes' $(\mathbb{R})$ is a separating family for $L_{c}^{p}(\mathbb{R})$ : recall that $\Re(z-x)^{-1} \in \mathcal{S}$ tieljes $(\mathbb{R})$ and hence $\Re(z-x)^{-2} \in \mathcal{S}$ tieljes' $(\mathbb{R})$. But, if for $f \in L_{c}^{p}(\mathbb{R})$ it holds that $\mathfrak{R}\left[\int(z-x)^{-2} f(x) d x\right]=0$ for all $z \in \mathbb{C} \backslash \mathbb{R}$ then $\mathfrak{R}\left[\int(z-x)^{-1} f(x) d x\right]=C$ for all $z \in \mathbb{C} \backslash \mathbb{R}$, and $C=0$ by taking $|z| \rightarrow \infty$. Hence, since on compact sets $\mathcal{S}$ tieljes $(\mathbb{R})$ uniformly approximates any polynomial and since the latter are dense in $L_{c}^{p}(\mathbb{R})$, we conclude that $f=0$. Thus, we can identify, for any $p \geqslant 1, L_{c}^{p}(\mathbb{R})$ as a subset of $\mathcal{X}$. In particular, we may and shall identify $F_{N}$ with $\left\{f^{\prime} \mapsto \int f^{\prime}(x) F_{N}(x) d x\right\} \in \mathcal{X}$.

Fix $a_{N} \rightarrow 0$ such that $N a_{N} \rightarrow \infty$. Combining [8, Theorem 4.6.9] and Theorem 5.1 we see that $\left\{a_{N}^{-1} F_{N}\right\}_{N}$ satisfies the LDP in $(\mathcal{X}, \mathcal{A})$, with speed $\left(N a_{N}\right)^{-2}$ and the good rate function $I(\cdot)$ of (1.3). By Lemma 1.3 we know that $I(\cdot)=\infty$ outside $L_{c}^{1, *}(\mathbb{R}) \subset L_{c}^{1}(\mathbb{R})$, so with $\left\{a_{N}^{-1} F_{N}\right\} \subset L_{c}^{1}(\mathbb{R})$, Theorem 1.1 follows from [8, Lemma 4.1.5(b)].

The following is an immediate corollary of Theorem 1.1 and Lemma 3.1:

COROLlary 5.2. - The conclusion of Theorem 1.1 continues to hold true when $F_{N}$ is replaced by $\bar{F}_{N}(x)=\mathbb{E} \hat{\mu}_{1}^{N}((-\infty, x])-\hat{\mu}_{1}^{N}((-\infty, x])$.

The advantage of working with $\bar{F}_{N}$ is that it allows us to strengthen the topology for which moderate deviations hold. For any $d<\infty$ and $\mathbf{f}=\left(f^{(1)}, \ldots, f^{(d)}\right) \in\left(\mathcal{C}_{b}^{1}(\mathbb{R})\right)^{d}$, define

$$
\bar{X}_{N}(\mathbf{f}):=\int \mathbf{f}^{\prime}(x) \bar{F}_{N}(x) d x=\operatorname{tr}_{N} \mathbf{f}\left(X_{N}\right)-\mathbb{E} \operatorname{tr}_{N} \mathbf{f}\left(X_{N}\right) \in \mathbb{R}^{d},
$$

by integration by parts.

Let $K^{\prime}=\left[k_{-}-1, k_{+}+1\right]$, where $K=\left[k_{-}, k_{+}\right]$is a compact interval containing the support of $\mu_{1}^{*}$. We now have the following approximation lemma whose proof is deferred: 
LeMma 5.3. - Assume $\mathbf{f}, \mathbf{f}_{m} \in\left(\mathcal{C}_{b}^{1}(\mathbb{R})\right)^{d}, d<\infty$, are such that

$$
\lim _{m \rightarrow \infty} \sup _{x \in K^{\prime}}\left|\partial_{x}\left(f^{(i)}-f_{m}^{(i)}\right)(x)\right|=0
$$

for $i=1, \ldots, d$. Then, for any $\delta>0$ and any $a_{N} \rightarrow 0$ with $N a_{N} \rightarrow \infty$,

$$
\limsup _{m \rightarrow \infty} \limsup _{N \rightarrow \infty}\left(N a_{N}\right)^{-2} \log \mathbb{P}\left(\left|\bar{X}_{N}(\mathbf{f})-\bar{X}_{N}\left(\mathbf{f}_{m}\right)\right|>\delta a_{N}\right)=-\infty .
$$

Proof of Theorem 1.2. - Fixing $a_{N} \rightarrow 0$ such that $N a_{N} \rightarrow \infty$ and $\mathbf{f} \in\left(\mathcal{C}_{b}^{1}(\mathbb{R})\right)^{d}$, there exists a sequence of functions $\mathbf{f}_{m} \in(\mathcal{S} \text { tieljes }(\mathbb{R}))^{d}$ for which (5.10) holds. Then, by (5.11), the random sequences $\left\{a_{N}^{-1} \bar{X}_{N}\left(\mathbf{f}_{m}\right)\right\}_{N}$ are exponentially good approximations of $\left\{a_{N}^{-1} \bar{X}_{N}(\mathbf{f})\right\}_{N}$ in $\mathbb{R}^{d}$. Recall Corollary 5.2, that $\left\{a_{N}^{-1} \bar{F}_{N}\right\}_{N}$ satisfies the LDP in $L_{c}^{1}(\mathbb{R})$ equipped with the $\mathcal{S}$ tieljes' $(\mathbb{R})$-topology, with speed $\left(N a_{N}\right)^{-2}$ and the good rate function $I(\cdot)$ of (1.3). By (5.9), $a_{N}^{-1} \bar{X}_{N}\left(\mathbf{f}_{m}\right)$ is for each $m$ the image of $a_{N}^{-1} \bar{F}_{N}$ under the map $F \mapsto$ $\int \mathbf{f}_{m}^{\prime}(x) F(x) d x: L_{c}^{1}(\mathbb{R}) \rightarrow \mathbb{R}^{d}$ which is continuous with respect to the $\mathcal{S}$ tieljes' $(\mathbb{R})$ topology. If $F \in L_{c}^{1}(\mathbb{R})$ has $I(F) \leqslant \alpha$ then $F / p_{1} \in L^{2}\left(\mu_{1}^{*}\right)$ with $\mu_{1}^{*}\left(\left(F / p_{1}\right)^{2}\right) \leqslant 2 \alpha$ (see (1.7)). By the Cauchy-Schwartz inequality in $L^{2}\left(\mu_{1}^{*}\right)$ and (5.10) we thus have that

$$
\sup \left\{\left|\int \mathbf{f}^{\prime}(x) F(x) d x-\int \mathbf{f}_{m}^{\prime}(x) F(x) d x\right|: I(F) \leqslant \alpha\right\} \leqslant \sqrt{2 \alpha} \mu_{1}^{*}\left(\left|\mathbf{f}^{\prime}-\mathbf{f}_{m}^{\prime}\right|^{2}\right)^{1 / 2} \rightarrow 0
$$

as $m \rightarrow \infty$ for each $\alpha<\infty$. Consequently, by [8, Theorem 4.2.23] it follows that $\left\{a_{N}^{-1} \bar{X}_{N}(\mathbf{f})\right\}_{N}$ satisfies the LDP in $\mathbb{R}^{d}$ with speed $\left(N a_{N}\right)^{-2}$ and the good rate function

$$
I_{\mathbf{f}}(\boldsymbol{y})=\inf \left\{I(F): F \in L_{c}^{1}(\mathbb{R}), \int \mathbf{f}^{\prime}(x) F(x) d x=\boldsymbol{y}\right\} .
$$

With $\mathcal{S}$ tieljes' $(\mathbb{R}) \subset \mathcal{C}_{b}^{\prime}(\mathbb{R})$ a separating family for $L_{c}^{1}(\mathbb{R})$, similarly to the proof of Theorem 1.1 we identify $L_{c}^{1}(\mathbb{R})$ as a subset of the algebraic dual $\overline{\mathcal{X}}$ of $\mathcal{C}_{b}^{\prime}(\mathbb{R})$, mapping $\bar{F}_{N}$ to $\left\{f^{\prime} \mapsto \int f^{\prime}(x) \bar{F}_{N}(x) d x\right\} \in \overline{\mathcal{X}}$. We equip $\overline{\mathcal{X}}$ with the $\mathcal{C}_{b}^{\prime}(\mathbb{R})$-topology and the corresponding cylinder $\sigma$-field. It then follows by [8, Theorem 4.6.9] and the above LDPs for $\left\{a_{N}^{-1} \bar{X}_{N}(\mathbf{f})\right\}_{N}$ that $\left\{a_{N}^{-1} \bar{F}_{N}\right\}_{N}$ satisfies the LDP in $\overline{\mathcal{X}}$, with speed $\left(N a_{N}\right)^{-2}$ and the good rate function

$$
\begin{aligned}
\hat{I}(G) & =\sup _{d<\infty} \sup _{\mathbf{f} \in\left(\mathcal{C}_{b}^{1}(\mathbb{R})\right)^{d}} I_{\mathbf{f}}\left(\left\langle\mathbf{f}^{\prime}, G\right\rangle\right) \\
& =\sup _{d<\infty} \sup _{\mathbf{f} \in\left(\mathcal{C}_{b}^{1}(\mathbb{R})\right)^{d}} \inf \left\{I(F): F \in L_{c}^{1}(\mathbb{R}), \int \mathbf{f}^{\prime}(x) F(x) d x=\left\langle\mathbf{f}^{\prime}, G\right\rangle\right\} .
\end{aligned}
$$

In particular, by (1.7),

$$
\begin{aligned}
\hat{I}(G) & \geqslant \sup _{g \in \mathcal{C}_{b}^{\prime}(\mathbb{R})} \inf \left\{\frac{\beta}{4} \mu_{1}^{*}\left(h^{2}\right): h \in L^{2}\left(\mu_{1}^{*}\right), \mu_{1}^{*}(g h)=\langle g, G\rangle\right\} \\
& =\frac{\beta}{4} \sup _{g \in \mathcal{C}_{b}^{\prime}(\mathbb{R})} \frac{\langle g, G\rangle^{2}}{\mu_{1}^{*}\left(g^{2}\right)} .
\end{aligned}
$$


Applying Lemma 6.2 for the function $\hat{I}(\cdot)$ and the vector space $\mathcal{W}=\mathcal{C}_{b}^{\prime}(\mathbb{R})$, we see that (5.13) implies that $\hat{I}(G)<\infty$ only if $G \in \overline{\mathcal{X}}$ belongs also to $L_{c}^{1, *}(\mathbb{R})$ (and moreover $\left.G / p_{1} \in L^{2}\left(\mu_{1}^{*}\right)\right)$. Consequently, with $\left\{a_{N}^{-1} \bar{F}_{N}\right\}_{N} \cup\{G: \hat{I}(G)<\infty\} \subset L_{c}^{1}(\mathbb{R})$, by $[8$, Lemma 4.1.5(b)] the LDP we obtained for $\left\{a_{N}^{-1} \bar{F}_{N}\right\}_{N}$ holds also within $L_{c}^{1}(\mathbb{R})$. Since $\left\langle\mathbf{f}^{\prime}, G\right\rangle=\int \mathbf{f}^{\prime}(x) G(x) d x$ for any $G \in L_{c}^{1}(\mathbb{R})$, considering $F=G$ in (5.12) we clearly see that $\hat{I}(G) \leqslant I(G)$. On the other hand, by [8, Lemma 4.6.5],

$$
I(G)=\sup _{d<\infty} \sup _{\mathbf{f} \in(\mathcal{S} \text { tieljes }(\mathbb{R}))^{d}} \inf \left\{I(F): F \in L_{c}^{1}(\mathbb{R}), \int \mathbf{f}^{\prime}(x) F(x) d x=\left\langle\mathbf{f}^{\prime}, G\right\rangle\right\},
$$

for any $G \in \mathcal{X}$, which in comparison with (5.12) shows that $\hat{I}(G) \geqslant I(G)$ for all $G \in L_{c}^{1}(\mathbb{R})$, completing the proof of the theorem.

Proof of Lemma 5.3. - We bring the proof in the real (symmetric) case, the Hermitian case being similar. By union of events bounds, it suffices to consider the case of $d=1$ in (5.11). To this end, set $\theta \in \mathcal{C}_{b}^{1}(\mathbb{R})$ such that $\theta(x)=1$ for all $x \in K$ and $\theta(x)=0$ for all $x \notin K^{\prime}$. Fixing $f, f_{m} \in \mathcal{C}_{b}^{1}(\mathbb{R})$ for which (5.10) holds, let $g_{m}(x):=\theta(x)\left(f(x)-f_{m}(x)\right)$, so that $\delta_{m}=\left\|g_{m}^{\prime}\right\|_{\infty} \rightarrow 0$ as $m \rightarrow \infty$. By (5.9) and [11, Theorem 1.1(b)], it then holds that for some $C>0$ and all $m, N, \delta>0$,

$$
\mathbb{P}\left(\left|\bar{X}_{N}\left(g_{m}\right)\right|>\delta a_{N}\right) \leqslant 2 \exp \left(-\frac{C \delta^{2}\left(N a_{N}\right)^{2}}{\delta_{m}^{2}}\right) .
$$

Since $\bar{X}_{N}(f)-\bar{X}_{N}\left(f_{m}\right)=\bar{X}_{N}\left(g_{m}\right)+\bar{X}_{N}\left(h_{m}\right)$ for $h_{m}(x)=(1-\theta(x))\left(f(x)-f_{m}(x)\right)$, it suffices to show that $\bar{X}_{N}\left(h_{m}\right)$ is exponentially negligible, i.e., that for any $m$ and $\delta>0$,

$$
\limsup _{N \rightarrow \infty}\left(N a_{N}\right)^{-2} \log \mathbb{P}\left(\left|\bar{X}_{N}\left(h_{m}\right)\right|>\delta a_{N}\right)=-\infty .
$$

To see (5.14), set $Y_{N}=N a_{N} \bar{X}_{N}\left(h_{m}\right)$ and $Z_{N}(\lambda)=\mathbb{E} \mathrm{e}^{\lambda Y_{N}}$. Denoting by $X_{i j}$ the $i j$ entry of the matrix $X_{N}$, we use a variant of Herbst's argument, similar to the proof of [11, Theorem 1.1(b)]. To this end, for $\lambda>0$, let

$$
G_{N}(\lambda):=\lambda^{2} Z_{N}(\lambda) \frac{d}{d \lambda}\left(\lambda^{-1} \log Z_{N}(\lambda)\right)=\mathbb{E}\left(\mathrm{e}^{\lambda Y_{N}} \log \left(\frac{\mathrm{e}^{\lambda Y_{N}}}{Z_{N}(\lambda)}\right)\right) .
$$

With $h_{m}$ a Lipschitz function, recall that

$$
\sum_{1 \leqslant i \leqslant j \leqslant N}\left(\partial_{X_{i j}} \operatorname{tr} h_{m}\left(X_{N}\right)\right)^{2} \leqslant 2 \operatorname{tr}_{N}\left(h_{m}^{\prime}\left(X_{N}\right)^{2}\right)
$$

(see [11, (2.12)]). Consequently, applying the logarithmic Sobolev inequality for the multivariate Gaussian distribution of $\left(X_{i j}, 1 \leqslant i \leqslant j \leqslant N\right)$ and the differentiable function $\exp \left(\lambda Y_{N} / 2\right)$, we have by (5.9) that for some universal constant $c<\infty$ and all $\lambda>0, N$, 


$$
\begin{aligned}
G_{N}(\lambda) & \leqslant c \mathbb{E}\left(\sum_{1 \leqslant i \leqslant j \leqslant N}\left(\partial_{X_{i j}} \mathrm{e}^{\lambda Y_{N} / 2}\right)^{2}\right) \\
& =c \lambda^{2}\left(N a_{N}\right)^{2} \mathbb{E}\left(\mathrm{e}^{\lambda Y_{N}} \sum_{1 \leqslant i \leqslant j \leqslant N}\left(\partial_{X_{i j}} \operatorname{tr}_{N} h_{m}\left(X_{N}\right)\right)^{2}\right) \\
& \leqslant 2 c \lambda^{2} a_{N}^{2} \mathbb{E}\left(\operatorname{tr}_{N}\left(h_{m}^{\prime}\left(X_{N}\right)^{2}\right) \mathrm{e}^{\lambda Y_{N}}\right) .
\end{aligned}
$$

With $h_{m}$ bounded, $Y_{N} \leqslant c_{m} N a_{N}$ for some $c_{m}<\infty$ and all $N$. Hence, for any $\lambda, \kappa>0$,

$$
\mathbb{E}\left(\operatorname{tr}_{N}\left(h_{m}^{\prime}\left(X_{N}\right)^{2}\right) \mathrm{e}^{\lambda Y_{N}}\right) \leqslant \frac{\kappa}{8} Z_{N}(\lambda)+\mathrm{e}^{\lambda c_{m} N a_{N}} \mathbb{P}\left(\operatorname{tr}_{N}\left(h_{m}^{\prime}\left(X_{N}\right)^{2}\right) \geqslant \frac{\kappa}{8}\right) .
$$

Since $h_{m}(x)=0$ for $x \in K$ that contains the support of $\mu_{1}^{*}$, clearly $\mu_{1}^{*}\left(\left(h_{m}^{\prime}\right)^{2}\right)=0$. Note that $\left(h_{m}^{\prime}\right)^{2} \in \mathcal{C}_{b}(\mathbb{R})$, so $\operatorname{tr}_{N}\left(h_{m}^{\prime}\left(X_{N}\right)^{2}\right)=\hat{\mu}_{X_{N}}^{N}\left(\left(h_{m}^{\prime}\right)^{2}\right) \rightarrow \mu_{1}^{*}\left(\left(h_{m}^{\prime}\right)^{2}\right)$ (see, e.g., [19]). With $\left\{\hat{\mu}_{X_{N}}^{N}\right\}_{N}$ exponentially tight at scale $N^{2}$ (see [1]), an application of [11, Theorem 1.1(b)] after truncating $\hat{\mu}_{X_{N}}^{N}$ to a large enough compact and uniformly approximating $\left(h_{m}^{\prime}\right)^{2}$ on this set by a Lipschitz function, reveals that also $\left|\hat{\mu}_{X_{N}}^{N}\left(\left(h_{m}^{\prime}\right)^{2}\right)-\mathbb{E} \hat{\mu}_{X_{N}}^{N}\left(\left(h_{m}^{\prime}\right)^{2}\right)\right| \rightarrow 0$, in fact with probability decaying exponentially in scale $N^{2}$, implying that

$$
\mathbb{P}\left(\left|\hat{\mu}_{X_{N}}^{N}\left(\left(h_{m}^{\prime}\right)^{2}\right)-\mu_{1}^{*}\left(\left(h_{m}^{\prime}\right)^{2}\right)\right| \geqslant \frac{\kappa}{8}\right) \leqslant \mathrm{e}^{-c N^{2}},
$$

for some $c>0$ (this last conclusion can also be seen directly by mimicking the argument for the upper bound in [6, Theorem 1.3 and Corollary 1.4], with initial condition $D_{N}$ ). With $a_{N} \rightarrow 0$ we thus deduce that for all $\varepsilon, \kappa>0, \lambda \in\left(0, \varepsilon^{-1} N\right]$ and $N>N_{0}(m, \kappa, \varepsilon)$,

$$
\mathbb{E}\left(\operatorname{tr}_{N}\left(h_{m}^{\prime}\left(X_{N}\right)^{2}\right) \mathrm{e}^{\lambda Y_{N}}\right) \leqslant \frac{\kappa}{4} Z_{N}(\lambda)
$$

Combining (5.15), (5.16) and (5.17) we see that for every $\kappa>0, \varepsilon>0$ and such $\lambda$ and $N$,

$$
\frac{d}{d \lambda}\left(\lambda^{-1} \log Z_{N}(\lambda)\right) \leqslant \frac{c \kappa a_{N}^{2}}{2}
$$

Since $Z_{N}(0)=1$ and $Z_{N}^{\prime}(0)=0$, it follows that $Z_{N}(\lambda) \leqslant \exp \left(c \kappa a_{N}^{2} \lambda^{2} / 2\right)$. Therefore, by Chebysheff's inequality,

$$
\mathbb{P}\left(\bar{X}_{N}\left(h_{m}\right)>\delta a_{N}\right)=\mathbb{P}\left(Y_{N}>\delta N a_{N}^{2}\right) \leqslant Z_{N}(\lambda) \mathrm{e}^{-\lambda \delta N a_{N}^{2}} \leqslant \mathrm{e}^{c \kappa a_{N}^{2} \lambda^{2} / 2-\lambda \delta N a_{N}^{2}} .
$$

Choosing $\varepsilon=c \kappa / \delta$ and $\lambda=\varepsilon^{-1} N$ it follows (applying the above once for $h_{m}$ and once for $-h_{m}$ ) that

$$
\limsup _{N \rightarrow \infty}\left(N a_{N}\right)^{-2} \log \mathbb{P}\left(\left|\bar{X}_{N}\left(h_{m}\right)\right|>\delta a_{N}\right) \leqslant-\frac{\delta^{2}}{2 \kappa c} .
$$

Since $\kappa$ is arbitrary, (5.14) follows, thus completing the proof of the lemma. 


\section{Free probability: properties of $\mathcal{V}_{1}(\cdot, \cdot)$ and $I(\cdot)$}

We have following [3] and [4], that if we let $\Delta_{s}$ be the differential operator on $\mathcal{C}^{1}(\mathbb{R})$ with values in $\mathcal{C}^{0}(\mathbb{R})$ given by

$$
\Delta_{s} f(x):=\int \frac{f(x)-f(y)}{x-y} d \mu_{s}^{*}(y),
$$

then (1.1) reads

$$
\partial_{s} f_{s}(x)=-\Delta_{s} \circ \partial_{x} f_{s}(x) .
$$

Let $\mathcal{M}(\mathbb{R})$ denote the space of finite, complex, Borel measures on $\mathbb{R}$. Consider the following vector subspaces of $\mathcal{C}^{k}(\mathbb{R}), k \geqslant 1$,

$$
\mathcal{G}_{k}:=\left\{\int \mathrm{e}^{\mathrm{i} \xi x} d \nu(\xi)+\sum_{p=1}^{n} a_{p} x^{p}: n<\infty, a_{p} \in \mathbb{C}, v \in \mathcal{M}(\mathbb{R}), \int|\xi|^{k} d|v|(\xi)<\infty\right\} .
$$

We let $\mathcal{G} \subset \mathcal{G}_{3}$ be the vector space of functions $g: \mathbb{R} \rightarrow \mathbb{C}$ for which a solution $f(x, s)=f_{s}(x) \in \mathcal{C}^{3,1}(\mathbb{R} \times[0,1])$ of (6.1) with time marginals $f_{s} \in \mathcal{G}_{3}$ and boundary condition $f_{1}(x)=g(x)$ exists. Recall that when $\Im(z)>0$,

$$
(z-x)^{-1}=\mathrm{i} \int_{\mathbb{R}_{+}} \mathrm{e}^{\mathrm{i} \xi(z-x)} d \xi \in \mathcal{G}_{3},
$$

with analogous expression for $(z-x)^{-1}$ when $\Im(z)<0$. Hence, $\mathcal{S}$ tieljes $(\mathbb{C}) \subset \mathcal{G}_{3}$, which as we have seen in (4.1) and (4.2) implies that $\mathcal{S}$ tieljes $(\mathbb{C}) \subset \mathcal{G}$. We next define

$$
\mathcal{V}_{1}(f, f):=\int_{0}^{1} \mu_{s}^{*}\left(\left|\partial_{x} f_{s}\right|^{2}\right) d s
$$

for any $f=f_{1} \in \mathcal{G}$. By our assumption that $\mu_{D}$ is compactly supported, there exists a compact set $K \subset\left\{x \in \mathbb{R}, d\left(x, \operatorname{supp}\left(\mu_{D}\right)\right) \leqslant 2\right\}$ that contains the support of $\mu_{s}^{*}$ for all $s \in[0,1]$. For any $f \in \mathcal{G}$, the continuous function $\partial_{x} f_{s}$ is uniformly bounded on the compact $K \times[0,1]$, implying that $\mathcal{V}_{1}(f, f)$ is finite.

We use free probability theory to prove the following approximation lemma.

LEMmA 6.1. - Let $h, g \in \mathcal{G}$. Then,

$$
\left|\mathcal{V}_{1}(h, h)^{1 / 2}-\mathcal{V}_{1}(g, g)^{1 / 2}\right| \leqslant \mu_{1}^{*}\left(\left|\partial_{x}(h-g)\right|^{2}\right)^{1 / 2}
$$

Proof. - Eq. (6.1) implies that whenever $f_{1} \in \mathcal{G}$,

$$
\partial_{s} \partial_{x} f_{s}(x)=\partial_{x} \partial_{s} f_{s}(x)=-\partial_{x} \circ \Delta_{s}\left(\partial_{x} f_{s}\right)(x) .
$$

Let $(\mathcal{A}, \tau)$ be a non-commutative probability space on which a free Brownian motion $S$. and a self-adjoint variable $D$ of law $\mu_{D}$, free with $S$ are defined. Recall that the operator 
$X_{s}$ with law $\mu_{s}^{*}$ can then be seen as the solution of the free differential equation

$$
d X_{t}=d S_{t}, \quad X_{0}=D .
$$

For $f \in \mathcal{G}_{1}$ consider the stochastic integral

$$
\begin{aligned}
\int_{0}^{t} \bar{\partial} f\left(X_{u}\right) \sharp d S_{u}:= & \mathrm{i} \int_{0}^{t} \int_{0}^{1} \int_{\mathbb{R}} \mathrm{e}^{\mathrm{i} \xi \alpha X_{u}} d S_{u} \mathrm{e}^{\mathrm{i} \xi(1-\alpha) X_{u} \xi d \nu(\xi) d \alpha} \\
& +\sum_{p=1}^{n} a_{p} \sum_{l=0}^{p-1} \int_{0}^{t} X_{u}^{l} d S_{u} X_{u}^{p-l-1}
\end{aligned}
$$

(cf. [3]). Then, for any $\psi(x, t)$ continuously differentiable with respect to $t$ and with time marginals $\psi(\cdot, t) \in \mathcal{G}_{2}$ we have that,

$\psi\left(X_{t}, t\right)=\psi\left(X_{0}, 0\right)+\int_{0}^{t} \bar{\partial} \psi\left(X_{u}, u\right) \sharp d S_{u}+\int_{0}^{t} \partial_{x} \circ \Delta_{u} \psi\left(X_{u}, u\right) d u+\int_{0}^{t} \partial_{t} \psi\left(X_{u}, u\right) d u$.

The formula for $\psi(x)$ that does not depend on time $t$ is derived in [3, p. 392] (apart from an erroneous factor of $1 / 2$ in the $d u$ term there). The generalization to $\psi(x, t)$ with smooth time dependence is then straight forward. Taking $f=f_{1} \in \mathcal{G}$ and applying (6.3) for $\psi(x, t)=\partial_{x} f_{t}(x)$, we find by (6.2) that

$$
\partial_{x} f_{t}\left(X_{t}\right)=\partial_{x} f_{0}\left(X_{0}\right)+\int_{0}^{t} \bar{\partial} \circ \partial_{x} f_{u}\left(X_{u}\right) \sharp d S_{u} .
$$

By [3, Proposition 3.2.3] it then follows that $t \mapsto \partial_{x} f_{t}\left(X_{t}\right):[0,1] \rightarrow(\mathcal{A}, \tau)$ is an $L^{2}$ martingale with respect to the filtration $\mathcal{A}_{t}$ generated by $D$ and $\left\{S_{u} ; u \leqslant t\right\}$, i.e., $\partial_{x} f_{t}\left(X_{t}\right)=\tau\left(\partial_{x} f_{1}\left(X_{1}\right) \mid \mathcal{A}_{t}\right)$ for any $t \in[0,1]$ with $\tau\left(\cdot \mid \mathcal{A}_{t}\right)$ the projection onto $\mathcal{A}_{t}$ in the non-commutative $L^{2}(\mathcal{A}, \tau)$ space (obtained by completion of $\mathcal{A}$ with respect to the norm $\left.\tau\left(|\cdot|^{2}\right)^{1 / 2}\right)$. Consequently, with $\bar{f}$ denoting the complex conjugate of $f$, for all $t \in[0,1]$,

$$
\begin{aligned}
\mu_{t}^{*}\left(\left|\partial_{x} f_{t}\right|^{2}\right) & =\tau\left(\partial_{x} f_{t}\left(X_{t}\right) \partial_{x} \bar{f}_{t}\left(X_{t}\right)\right)=\tau\left(\tau\left(\partial_{x} f_{1}\left(X_{1}\right) \mid \mathcal{A}_{t}\right) \tau\left(\partial_{x} \bar{f}_{1}\left(X_{1}\right) \mid \mathcal{A}_{t}\right)\right) \\
& \leqslant \tau\left(\partial_{x} f_{1}\left(X_{1}\right) \partial_{x} \bar{f}_{1}\left(X_{1}\right)\right)=\mu_{1}^{*}\left(\left|\partial_{x} f\right|^{2}\right),
\end{aligned}
$$

and therefore

$$
\mathcal{V}_{1}(f, f) \leqslant \mu_{1}^{*}\left(\left|\partial_{x} f\right|^{2}\right) .
$$

Fix $h, g \in \mathcal{G}$ and apply this inequality for $f=h-g \in \mathcal{G}$, to get

$$
\mathcal{V}_{1}(h, h)^{1 / 2} \leqslant \mathcal{V}_{1}(g, g)^{1 / 2}+\mathcal{V}_{1}(h-g, h-g)^{1 / 2} \leqslant \mathcal{V}_{1}(g, g)^{1 / 2}+\mu_{1}^{*}\left(\left|\partial_{x}(h-g)\right|^{2}\right)^{1 / 2}
$$

which completes the proof.

The next lemma is a key ingredient in the proof of Lemma 1.3. 
LEMMA 6.2. - Let $\mathcal{W} \subset \mathcal{C}_{b}(\mathbb{R})$ be a vector space that separates points in $L_{c}^{1}(\mathbb{R})$ and is dense in $L^{2}\left(\mu_{1}^{*}\right)$. Suppose that

$$
I(F) \geqslant \frac{\beta}{4} \sup _{g \in \mathcal{W}} \frac{\langle g, F\rangle^{2}}{\mu_{1}^{*}\left(g^{2}\right)}
$$

for every $F \in \mathcal{W}^{\prime}$ (the algebraic dual of $\mathcal{W}$ ). Then, $I(\cdot)$ is finite only for $F \in L_{c}^{1, *}(\mathbb{R})$, in which case (1.7) holds.

Proof. - Let $\mathcal{H}$ be the Hilbert space equipped with the scalar product $(h, g)=\mu_{1}^{*}(h g)$ constructed by taking the quotient of $\mathcal{W}$ by the equivalence relation $(h, h)=0$ and completed for the norm $\|\cdot\|_{L^{2}\left(\mu_{1}^{*}\right)}$. Then, the inequality (6.4) shows that for any $F$ such that $I(F)<\infty$, the linear map $g \mapsto\langle g, F\rangle: \mathcal{W} \rightarrow \mathbb{R}$ has operator norm of at most $\sqrt{2 I(F)}<\infty$ for the $\|\cdot\|_{L^{2}\left(\mu_{1}^{*}\right)}$-norm, hence can be extended continuously to $\mathcal{H}$. Thus, by Riesz's theorem there exists $h \in \mathcal{H}$ such that

$$
\langle g, F\rangle=\mu_{1}^{*}(h g)=\int g(x)\left(h p_{1}(x)\right) d x, \quad \forall g \in \mathcal{H} .
$$

Further, if $(g, g)=0$ for some $g \in \mathcal{W}$ then we find directly from (6.4) that $\langle g, F\rangle=0$ for otherwise $I(F)=\infty$. We conclude that there exists an $h \in \mathcal{H}$ such that $\langle g, F\rangle=$ $\int g(x)\left(h p_{1}(x)\right) d x$ for all $g \in \mathcal{W}$.

Letting $\widetilde{F}(x)=h p_{1}(x)$, we deduce that $\langle g, F\rangle=\int \widetilde{F}(x) g(x) d x$ and, since $p_{1}$ is compactly supported (see [2]) and $h \in L^{2}\left(\mu_{1}^{*}\right)$, it follows that $\widetilde{F}$ belongs to $L_{c}^{1, *}(\mathbb{R})$. Since $\mathcal{W}$ separates points in $L_{c}^{1}(\mathbb{R})$ we may and shall identify $\widetilde{F}$ with $g \mapsto \int \widetilde{F} g d x \in \mathcal{W}^{\prime}$, hence, identifying in the sequel $\widetilde{F}$ and $F$. Further, with $\mathcal{W}$ being a dense subset of $L^{2}\left(\mu_{1}^{*}\right)$,

$$
I(F) \geqslant \frac{\beta}{4} \sup _{g \in \mathcal{W}} \frac{\langle g, F\rangle^{2}}{\mu_{1}^{*}\left(g^{2}\right)}=\frac{\beta}{4} \mu_{1}^{*}\left(h^{2}\right)=\frac{\beta}{4} \int \frac{F^{2}(x)}{p_{1}(x)} d x,
$$

proving the right inequality in (1.7). The left inequality in (1.7) is an immediate consequence of the Cauchy-Schwartz inequality in $L^{2}\left(\mu_{1}^{*}\right)$.

Proof of Lemma 1.3. - To simplify the notations, we only consider the case $\beta=2$. Let $S=S_{1}$ be a semicircular variable and self-adjoint $D$ of law $\mu_{D}$ free with $S$, defined on the non-commutative probability space $(\mathcal{A}, \tau)$. Let $\mathbb{C}\langle D, S\rangle$ denote the set of polynomial functions in $D$ and $S$ with complex valued coefficients, with $\mathbb{C}\langle S+D\rangle(\mathbb{R}\langle S+D\rangle)$, denoting the subset of polynomials in $S+D$, with complex (respectively, real) valued coefficients.

Noting that the set of polynomial functions is closed with respect to the operator $\Delta_{s}$, which reduces the degree of the polynomial, it is proved in [9] that $\mathbb{C}\langle S+D\rangle \subset \mathcal{G}$. Moreover, an explicit non-negative operator $\Xi: \mathbb{C}\langle D, S\rangle \mapsto \mathbb{C}\langle D, S\rangle$ is constructed there, such that $\tau(P \Xi P) \geqslant 0$ for any $P \in \mathbb{C}\langle D, S\rangle$, while for any $P \in \mathbb{C}\langle S+D\rangle$,

$$
\begin{aligned}
& \mathcal{V}_{1}(P, P)=\tau\left(P^{\prime}\left[(I+\Xi)^{-1} P^{\prime}\right]\right), \\
& \tau(P(S+D)(I+\Xi) P(S+D))
\end{aligned}
$$




$$
=\mu_{1}^{*}\left(P^{2}\right)+\int\left(\frac{P(x)-P(y)}{x-y}\right)^{2} d \mu_{1}^{*}(x) d \mu_{1}^{*}(y) .
$$

We assumed that $\mu_{D}$ is compactly supported, hence so is $\mu_{1}^{*}$ and we can approximate, in view of Weierstrass theorem, any function $h \in \mathcal{S}$ tieljes $(\mathbb{R})$ by polynomial functions $P_{n}^{h}$ such that

$$
\mu_{1}^{*}\left(\left(\partial_{x}\left(h-P_{n}^{h}\right)\right)^{2}\right) \leqslant n^{-2}
$$

By Lemma 6.1 and (6.5), then

$$
\begin{aligned}
\mathcal{V}_{1}(h, h)=\lim _{n \rightarrow \infty} \mathcal{V}_{1}\left(P_{n}^{h}, P_{n}^{h}\right) & =\lim _{n \rightarrow \infty} \tau\left(\left(\partial_{x} P_{n}^{h}\right)\left[(I+\Xi)^{-1}\left(\partial_{x} P_{n}^{h}\right)\right]\right) \\
& \leqslant \limsup _{n \rightarrow \infty} \tau\left(\left(\partial_{x} P_{n}^{h}\right)^{2}\right) \\
& =\limsup _{n \rightarrow \infty} \mu_{1}^{*}\left(\left(\partial_{x} P_{n}^{h}\right)^{2}\right)=\mu_{1}^{*}\left(\left(\partial_{x} h\right)^{2}\right),
\end{aligned}
$$

where we have used the fact that $\Xi: \mathbb{C}\langle D, S\rangle \mapsto \mathbb{C}\langle D, S\rangle$ is non-negative. Consequently,

$$
\begin{aligned}
I(F) & =\sup _{h \in \mathcal{S} \text { tieljes }(\mathbb{R})}\left\{\left\langle h^{\prime}, F\right\rangle-\frac{1}{2} \mathcal{V}_{1}(h, h)\right\} \\
& \geqslant \sup _{h \in \mathcal{S} \text { tieljes }(\mathbb{R})}\left\{\left\langle h^{\prime}, F\right\rangle-\frac{1}{2} \mu_{1}^{*}\left(\left(h^{\prime}\right)^{2}\right)\right\}=\sup _{h \in \mathcal{S} \text { tieljes }(\mathbb{R})}\left\{\frac{\left\langle h^{\prime}, F\right\rangle^{2}}{2 \mu_{1}^{*}\left(\left(h^{\prime}\right)^{2}\right)}\right\} .
\end{aligned}
$$

The vector space $\mathcal{W}=\mathcal{S}$ tieljes' $(\mathbb{R}) \subset \mathcal{C}_{b}(\mathbb{R})$ is dense in $L^{2}\left(\mu_{1}^{*}\right)$. Fixing a linear functional $F$ on $\mathcal{W}$, we thus deduce from (6.8) and Lemma 6.2 that $I(F)<\infty$ only for $F \in L_{c}^{1, *}(\mathbb{R})$, in which case the inequalities of (1.7) hold.

We turn to prove that $I(F) \leqslant J(F)$ for all $F \in \mathcal{P}$. To this end, fix $h \in \mathcal{S}$ tieljes $(\mathbb{R})$. Taking the polynomial function $P=P_{n}^{h}$ of (6.7), we find by Lemma 6.1 that for any $f \in L^{2}\left(\mu_{1}^{*}\right)$,

$$
\begin{aligned}
\left\langle h^{\prime}, f p_{1}\right\rangle-\frac{1}{2} \mathcal{V}_{1}(h, h) \leqslant & \left\langle P^{\prime}, f p_{1}\right\rangle-\frac{1}{2} \mathcal{V}_{1}(P, P)+n^{-1}\left(\int f^{2}(x) p_{1}(x) d x\right)^{1 / 2} \\
& +n^{-1}\left(\mathcal{V}_{1}(P, P)^{1 / 2}+\mathcal{V}_{1}(h, h)^{1 / 2}\right) \\
\leqslant & \left\langle P^{\prime}, f p_{1}\right\rangle-\frac{1}{2} \mathcal{V}_{1}(P, P)+n^{-1}\|f\|_{L^{2}\left(\mu_{1}^{*}\right)} \\
& +n^{-1}\left(n^{-1}+2 \mathcal{V}_{1}(h, h)^{1 / 2}\right)
\end{aligned}
$$

Considering $n \rightarrow \infty$ we see that for any $h \in \mathcal{S}$ tieljes $(\mathbb{R})$,

$$
\left\langle h^{\prime}, f p_{1}\right\rangle-\frac{1}{2} \mathcal{V}_{1}(h, h) \leqslant \sup _{P \in \mathbb{R}\langle S+D\rangle}\left\{\left\langle P^{\prime}, f p_{1}\right\rangle-\frac{1}{2} \mathcal{V}_{1}(P, P)\right\} .
$$

By the non-negativity of $\Xi$, (6.5) and (6.6), it follows that for any $h, Q \in \mathbb{R}\langle S+D\rangle$,

$$
\begin{aligned}
& \left(\int\left(h^{\prime} Q p_{1}\right)(x) d x\right)^{2} \\
& =\left(\tau\left(h^{\prime}(S+D) Q(S+D)\right)\right)^{2}
\end{aligned}
$$




$$
\begin{aligned}
& \leqslant \tau\left(h^{\prime}(I+\Xi)^{-1} h^{\prime}\right) \tau(Q(I+\Xi) Q) \\
& =\mathcal{V}_{1}(h, h)\left[\mu_{1}^{*}\left(Q^{2}\right)+\int\left(\frac{Q(x)-Q(y)}{x-y}\right)^{2} d \mu_{1}^{*}(x) d \mu_{1}^{*}(y)\right] .
\end{aligned}
$$

Therefore, by (6.9) and (6.10), it follows that

$$
\begin{aligned}
I\left(Q p_{1}\right) & \leqslant \sup _{P \in \mathbb{R}\langle S+D\rangle}\left\{\left\langle P^{\prime}, Q p_{1}\right\rangle-\frac{1}{2} \mathcal{V}_{1}(P, P)\right\} \\
& =\frac{1}{2} \sup _{h \in \mathbb{R}\langle S+D\rangle} \frac{\left(\int\left(h^{\prime} Q p_{1}\right)(x) d x\right)^{2}}{\mathcal{V}_{1}(h, h)} \\
& \leqslant \frac{1}{2}\left[\mu_{1}^{*}\left(Q^{2}\right)+\int\left(\frac{Q(x)-Q(y)}{x-y}\right)^{2} d \mu_{1}^{*}(x) d \mu_{1}^{*}(y)\right]=J\left(Q p_{1}\right) .
\end{aligned}
$$

Equipped with (6.11), let $Q^{\delta}$ be the polynomial functions appearing in the definition of $F=f p_{1} \in \mathcal{P}$. Then, by (6.11) and the lower semi-continuity of $f \mapsto$ $I\left(f p_{1}\right): L^{2}\left(\mu_{1}^{*}\right) \rightarrow \mathbb{R}$

$$
I\left(f p_{1}\right) \leqslant \liminf _{\delta \rightarrow 0} I\left(Q^{\delta} p_{1}\right) \leqslant \liminf _{\delta \rightarrow 0} J\left(Q^{\delta} p_{1}\right) \leqslant J\left(f p_{1}\right),
$$

as stated. In particular, we have that for $f \in \mathcal{C}_{b}^{1}(\mathbb{R})$,

$$
J\left(f p_{1}\right)=\frac{1}{2} \int f^{2} d \mu_{1}^{*}+\frac{1}{2} \iint\left(\int_{0}^{1} f^{\prime}(\alpha x+(1-\alpha) y) d \alpha\right)^{2} d \mu_{1}^{*}(x) d \mu_{1}^{*}(y)
$$

(see (1.6)). For such $f$, by Weierstrass theorem there exist polynomials $Q^{\delta}$ such that $\partial_{x}\left(Q^{\delta}-f\right) \rightarrow 0$ uniformly on the compact, convex hull of the support of $\mu_{1}^{*}$, hence also $J\left(Q^{\delta} p_{1}\right) \rightarrow J\left(f p_{1}\right)$, implying that $f p_{1} \in \mathcal{P}$ and completing the proof of the lemma.

Proof of Lemma 1.4. - Again, up to multiplying the rate function by $2^{-1} \beta$, we can only consider the case $\beta=2$. For $h \in \mathcal{C}_{b}^{2}([-2,2])$, let

$$
A(h):=-\frac{1}{4 \pi^{2}} \int_{-2}^{2} \int_{-2}^{2} \frac{h(x) h^{\prime}(y)}{y-x} \frac{\sqrt{4-y^{2}}}{\sqrt{4-x^{2}}} d y d x
$$

and recall that by [14, Theorem 2.4] this is half the asymptotic variance in the CLT for the spectral measure of Wigner matrices $W_{N}$. Consequently, in view of (1.4) we see that (1.8) holds when $\mu_{D}=0$.

We provide instead a direct proof which is also the key to showing that $I(\cdot)=J(\cdot)$. Recall that when $\mu_{D}=0$, then $\mu_{1}^{*}(d y)=\sigma_{1}(d y)=(2 \pi)^{-1} \sqrt{4-y^{2}} 1_{|y| \leqslant 2} d y$ is the well known semi-circle law. Our starting point is [9, Remark (6.2)], where it is shown that $\mathcal{V}_{1}(h, h)=\sigma_{1}\left(h^{\prime}\left[(I+\Xi)^{-1} h^{\prime}\right]\right)$ for polynomial functions $h$, with $\Xi$ the integral operator with domain $\mathcal{D} \supset \mathcal{C}_{b}^{2}([-2,2])$, such that for $|x| \leqslant 2$ and $f \in \mathcal{C}_{b}^{2}([-2,2])$, 


$$
\begin{aligned}
\Xi f(x) & :=2 P V \int \frac{f(x)-f(y)}{(x-y)^{2}} \sigma_{1}(d y) \\
& =x f^{\prime}(x)-2 \int_{-2}^{2} \int_{0}^{1} \int_{0}^{1} \alpha f^{\prime \prime}(\eta \alpha y+(1-\eta \alpha) x) d \alpha d \eta \sigma_{1}(d y),
\end{aligned}
$$

where $P V$ stands for Cauchy's principal value, and the second line follows from the identity

$$
P V \int(x-y)^{-1} \sigma_{1}(d y)=0.5 x \quad \forall|x| \leqslant 2,
$$

(see, e.g., [16, p. 74]), and the fact that

$$
\int_{0}^{1} \int_{0}^{1} \alpha f^{\prime \prime}(\eta \alpha y+(1-\eta \alpha) x) d \alpha d \eta=\frac{f(y)-f(x)-(y-x) f^{\prime}(x)}{(x-y)^{2}} .
$$

In particular, we see that $\Xi f \in \mathcal{C}_{b}([-2,2])$ when $f \in \mathcal{C}_{b}^{2}([-2,2])$. Let $\mathcal{C}_{b}^{1}\left(\sigma_{1}\right)$ denote the subset of $g \in \mathcal{C}_{b}([-2,2])$, such that $\psi_{g}^{\prime}(y):=\partial_{y}\left(g(y) \sqrt{4-y^{2}}\right) \in L^{1}((-2,2))$. As already noted in [9, Remark (6.2)], integrating by parts the first line of (6.12) and using the fact that

$$
P V \int_{-2}^{2}(x-y)^{-1} \partial_{y}\left(\sqrt{4-y^{2}}\right) d y=\pi
$$

for $x \in(-2,2)$ (see [18, Eq. (6), p. 174]), we obtain the following formula, valid for all $g \in \mathcal{C}_{b}^{1}\left(\sigma_{1}\right)$,

$$
[(I+\Xi) g](x)=\frac{1}{\pi} P V \int_{-2}^{2} \frac{\psi_{g}^{\prime}(y)}{x-y} d y:=\hat{h}^{\prime}(x), \quad-2<x<2 .
$$

By [18, p. 178], for any given $\hat{h} \in \mathcal{C}_{b}^{2}([-2,2])$, Eq. (6.15) has a unique solution $\psi_{g}^{\prime}(\cdot) \in L^{1}((-2,2))$ such that $\int_{-2}^{2} \psi_{g}^{\prime}(x) d x=0$. This solution is given by

$$
\psi_{g}^{\prime}(x)=\frac{1}{\pi} P V \int_{-2}^{2} \frac{\sqrt{4-y^{2}}}{\sqrt{4-x^{2}}} \frac{\hat{h}^{\prime}(y)}{y-x} d y .
$$

We thus see that for all $\hat{h} \in \mathcal{C}_{b}^{2}([-2,2]), g=(I+\Xi)^{-1} \hat{h}^{\prime} \in \mathcal{C}_{b}^{1}\left(\sigma_{1}\right)$ is well defined and satisfies $g(x)=\left(4-x^{2}\right)^{-1 / 2} \int_{-2}^{x} \psi_{g}^{\prime}(t) d t$. Moreover, we have from (6.15), (6.16) and integration by parts, that for $\hat{h}$ as above,

$$
\sigma_{1}\left(\hat{h}^{\prime}\left[(I+\Xi)^{-1} \hat{h}^{\prime}\right]\right)=\frac{1}{2 \pi} \int_{-2}^{2} \hat{h}^{\prime}(x) \psi_{g}(x) d x=-\frac{1}{2 \pi} \int_{-2}^{2} \hat{h}(x) \psi_{g}^{\prime}(x) d x=2 A(\hat{h}) .
$$


Using once more (6.14) resulting with $P V \int(x-y)^{-1}\left(\sqrt{4-y^{2}}\right)^{-1} d y=0$ for $|x| \leqslant 2$, we note that for any $\hat{h} \in \mathcal{C}_{b}^{2}([-2,2])$,

$$
\begin{aligned}
A(\hat{h}) & =-\frac{1}{4 \pi^{2}} \int_{-2}^{2} \int_{-2}^{2} \hat{h}^{\prime}(y) \frac{(\hat{h}(x)-\hat{h}(y))}{y-x} \frac{\sqrt{4-y^{2}}}{\sqrt{4-x^{2}}} d y d x \\
& =\frac{1}{4 \pi^{2}} \int_{-2}^{2} \int_{-2}^{2} \hat{h}^{\prime}(y) \int_{0}^{1} \hat{h}^{\prime}(\alpha x+(1-\alpha) y) d \alpha \frac{\sqrt{4-y^{2}}}{\sqrt{4-x^{2}}} d y d x .
\end{aligned}
$$

Since $\int_{-2}^{2}\left(4-x^{2}\right)^{-1 / 2} d x<\infty$, it is not hard to see, from (6.17) and (6.18), that $\sigma_{1}\left(Q_{n}^{\prime}\left[(I+\Xi)^{-1} Q_{n}^{\prime}\right]\right)$ converges to $\sigma_{1}\left(h^{\prime}\left[(I+\Xi)^{-1} h^{\prime}\right]\right)$ for any $h \in \mathcal{C}_{b}^{2}(\mathbb{R})$ and polynomial functions $Q_{n}$ such that $Q_{n}$ and $Q_{n}^{\prime}$ approximate $h$ and $h^{\prime}$ uniformly on [-2,2]. By Lemma 6.1, $\mathcal{V}_{1}(h, h)=\lim _{n} \mathcal{V}_{1}\left(Q_{n}, Q_{n}\right)$ as soon as $Q_{n}^{\prime}$ approximate uniformly $h^{\prime}$ on $[-2,2]$ (being the support of $\mu_{1}^{*}$ ), so in particular the equality $\mathcal{V}_{1}(h, h)=$ $\sigma_{1}\left(h^{\prime}\left[(I+\Xi)^{-1} h^{\prime}\right]\right)=2 A(h)$ extends to all $h \in \mathcal{S}$ tieljes $(\mathbb{R})$, resulting with (1.8).

Turning to prove (1.9), fix $f \in \mathcal{C}_{b}^{3}([-2,2])$ and $h \in \mathcal{S}$ tieljes $(\mathbb{R})$. Then, $\hat{h}^{\prime}:=h^{\prime}-(I+$ $\Xi) f \in \mathcal{C}_{b}^{1}([-2,2])$ and $g:=(I+\Xi)^{-1} h^{\prime} \in \mathcal{C}_{b}^{1}\left(\sigma_{1}\right)$ exists, hence by $(6.17)$,

$$
\begin{aligned}
2 A(\hat{h})= & \sigma_{1}((g-f)[(I+\Xi)(g-f)]) \\
= & \sigma_{1}(f[(I+\Xi) f])+\sigma_{1}\left(h^{\prime}\left[(I+\Xi)^{-1} h^{\prime}\right]\right)-\sigma_{1}(g[(I+\Xi) f]) \\
& -\sigma_{1}(f[(I+\Xi) g]) .
\end{aligned}
$$

From the definition of $\Xi$ in (6.12), we also have that $I+\Xi$ is a symmetric non-negative operator on the functions $f$ and $g$ considered here, with

$$
\begin{aligned}
\sigma_{1}(g[(I+\Xi) f])= & \int g(x) f(x) \sigma_{1}(d x) \\
& +\iint \frac{(g(x)-g(y))}{(x-y)} \frac{(f(x)-f(y))}{(x-y)} \sigma_{1}(d y) \sigma_{1}(d x) \\
= & \sigma_{1}(f[(I+\Xi) g]) .
\end{aligned}
$$

So, with $0 \leqslant \sigma_{1}((g-f)[(I+\Xi)(g-f)])<\infty$, we have that

$$
A(\hat{h})=\frac{1}{2} \sigma_{1}(f[(I+\Xi) f])+\frac{1}{2} \sigma_{1}\left(h^{\prime}\left[(I+\Xi)^{-1} h^{\prime}\right]\right)-\sigma_{1}\left(f h^{\prime}\right) \geqslant 0 .
$$

Since $\mathcal{S}$ tieljes' $(R)$ is dense in $\mathcal{C}_{b}^{1}([-2,2])$, approximating $\partial_{x}((I+\Xi) f)$ uniformly on $[-2,2]$ by a sequence $h_{n}^{\prime \prime}$, with $h_{n} \in \mathcal{S}$ tieljes $(\mathbb{R})$ (also approximating uniformly the function $(I+\Xi) f$ and its primitive), it follows from (6.18) that then $A\left(\hat{h}_{n}\right) \rightarrow 0$. We thus deduce from (6.20) that for $F=f p_{1}$ with $f \in \mathcal{C}_{b}^{3}([-2,2])$,

$$
\begin{aligned}
I(F) & :=\sup _{h \in \mathcal{S} \text { tieljes }(\mathbb{R})}\left\{\left\langle h^{\prime}, F\right\rangle-\frac{1}{2} \mathcal{V}_{1}(h, h)\right\} \\
& =\sup _{h \in \mathcal{S} \text { tieljes }(\mathbb{R})}\left\{\sigma_{1}\left(h^{\prime} f\right)-\frac{1}{2} \sigma_{1}\left(h^{\prime}\left[(I+\Xi)^{-1} h^{\prime}\right]\right)\right\}
\end{aligned}
$$




$$
\begin{aligned}
& =\frac{1}{2} \sigma_{1}(f[(I+\Xi) f])-\inf _{h \in \mathcal{S} \text { tieljes }(\mathbb{R})} A(\hat{h})=\frac{1}{2} \sigma_{1}(f[(I+\Xi) f]) \\
& =\int_{-2}^{2}\left\{P V \int_{-2}^{2} \frac{F^{\prime}(y)}{(x-y)} d y\right\} F(x) d x,
\end{aligned}
$$

where the last line comes from (6.15). Integrating by parts finally gives

$$
I(F)=-\int_{-2}^{2} \int_{-2}^{2} F^{\prime}(y) F^{\prime}(x) \log |x-y| d x d y .
$$

\section{Acknowledgement}

We thank Dan Voiculescu for helpful discussions concerning the non-commutative Fisher information.

\section{REFERENCES}

[1] G. Ben Arous, A. Guionnet, Large deviations for Wigner's law and Voiculescu's noncommutative entropy, Probab. Theory Related Fields 108 (1997) 517-542.

[2] P. Biane, On the free convolution with semi-circular distribution, Indiana Univ. Math. J. 46 (1997) 705-718.

[3] P. Biane, R. Speicher, Stochastic calculus with respect to free Brownian motion and analysis on Wigner space, Probab. Theory Related Fields 112 (1998) 373-409.

[4] P. Biane, R. Speicher, Free diffusions, free entropy and free Fisher information, Ann. Inst. H. Poincaré Probab. Statist. 37 (2001) 581-606.

[5] T. Cabanal-Duvillard, Fluctuations de la loi empirique des grandes matrices aléatoires, Ann. Inst. H. Poincaré Probab. Statist. 37 (2001) 373-402.

[6] T. Cabanal-Duvillard, A. Guionnet, Large deviations upper bounds and non-commutative entropies for some matrices ensembles, Ann. Probab. 29 (2001) 1205-1261.

[7] T. Cabanal-Duvillard, A. Guionnet, Discussion around non-commutative entropies, Adv. Math. (2002), submitted for publication.

[8] A. Dembo, O. Zeitouni, Large Deviations Techniques and Applications, Second Edition, Springer, New York, 1998.

[9] A. Guionnet, Large deviation upper bounds and central limit theorems for band matrices and non-commutative functionnals of Gaussian large random matrices, Ann. Inst. H. Poincaré Probab. Statist. 38 (2002) 341-384.

[10] A. Guionnet, First order asymptotic of matrix integrals; a rigorous approach towards the understanding of matrix models, Preprint, 2002.

[11] A. Guionnet, O. Zeitouni, Concentration of the spectral measure for large matrices, Electron. Comm. Probab. 5 (2000) 119-136.

[12] A. Guionnet, O. Zeitouni, Large deviation asymptotics for spherical integrals, J. Funct. Anal. 188 (2002) 461-515.

[13] S. Israelsson, Asymptotic fluctuations of a particle system with singular interaction, Stochastic Process. Appl. 93 (2001) 25-56.

[14] K. Johansson, On fluctuations of eigenvalues of random Hermitian matrices, Duke Math. J. 91 (1998) 151-204. 
[15] C. Kipnis, S. Olla, S.R.S. Varadhan, Hydrodynamics and large deviation for simple exclusion processes, Comm. Pure Appl. Math. 42 (1989) 115-137.

[16] M.L. Mehta, Random Matrices, Second Edition, Academic Press, 1991.

[17] A. Puhalskii, The method of stochastic exponentials for large deviations, Stochastic Process. Appl. 54 (1994) 45-70.

[18] F. Tricomi, Integral Equations, Interscience, 1970.

[19] D. Voiculescu, Lectures on free probability theory, in: Lecture Notes in Math., Vol. 1738, 2000, pp. 283-349.

[20] E. Wigner, On the distribution of the roots of certain symmetric matrices, Ann. Math. 67 (1958) 325-327.

[21] L.-M. Wu, Large deviations, moderate deviations and LIL for empirical processes, Ann. Probab. 22 (1994) 17-27. 